\title{
Firing-rate, symbolic dynamics and frequency dependence in periodically driven spiking models: a piecewise-smooth approach*
}

\author{
Albert Granados ${ }^{\dagger 1}$ and Maciej Krupa ${ }^{\ddagger 2}$ \\ ${ }^{1,2}$ Inria Paris-Rocquencourt
}

\begin{abstract}
In this work we consider a periodically forced generic integrate-and-fire model with a unique attracting equilibrium in the subthreshold dynamics and study the dependence of the firing-rate on the frequency of the drive. In an earlier study we have obtained rigorous results on the bifurcation structure in such systems, with emphasis on the relation between the firing-rate and the rotation number of the existing periodic orbits. In this work we study how these bifurcation structures behave upon variation of the frequency of the input. This allows us to show that the dependence of the firing-rate on frequency of the drive follows a devil's staircase with non-monotonic steps and that there is an optimal response in the whole frequency domain. We also characterize certain bounded frequency windows in which the firing-rate exhibits a bell-shaped envelope with a global maximum.
\end{abstract}

\section{Introduction}

In this work we study periodically driven excitable systems of integrate and fire type, widely used to model the dynamics of the membrane potential of a neuron. We assume that the periodic forcing term, or the external input, satisfies a constraint which we refer to as dose conservation. The constraint is defined as fixing the total amount (cumulated dose) in a given time (observation time). As argued in $\$ 2$, this is equivalent to fixing the average rate of the cell stimulus, for example the applied current, the amount of neurotransmitter, or the amount of hormone per time unit, respectively. One of the goals of this work is to prove

*This work has been financially supported by the Large Scale Initiative Action REGATE, by the Spanish MINECO-FEDER Grants MTM2009-06973, MTM2012-31714 and the Catalan Grant 2009SGR859.

†albert.granados@inria.fr

${ }^{\ddagger}$ maciej.krupa@inria.fr 
that the system exhibits an optimal response, in terms of the firing-rate, which can be achieved by tuning the system parameters, like period or amplitude, to obtain the maximal firing-rate of the system (the average number of spikes per unit time). In particular, we consider square wave input and focus on the variation of the period while either the amplitude or the duration of the pulse is fixed.

The mathematical content of our study is to investigate the bifurcation structure of periodic orbits, as they completely determine the dynamics for the class of systems we study. In contrast to other studies KHR81, CB99, CO00, Coo01, COS01, CTW12, TB08, TB09, LC05, JMB ${ }^{+} 13$, which use Poincaré maps, our approach is by means of a stroboscopic map. This map is discontinuous, but, for most parameter values, it has the advantage of being contracting on the continuous components. Hence, as shown in GKC13, results in non-smooth systems can be applied to get a complete description of periodic orbits and their rotation numbers. In this work we use this information to understand the behaviour of the firing-rate under frequency variation of the drive. In particular, we prove the existence of an optimal response corresponding to the maximal firing-rate.

The setting we have chosen for this paper is very simple from the biological point of view, but it has the advantage of being mathematically tractable. This is mainly given by assuming that the unforced system possesses a unique attracting point in the subthreshold dynamics. Even simple generalizations, for example allowing the system to undergo a subthreshold saddle-node bifurcation, lead to complications, as the stroboscopic map can be expansive, so that the existence of a globally stable attractor cannot be expected. In particular it is not clear if the firing-rate can be uniquely defined in the context of such generalizations, as well as how to obtain rigorous results about it.

The main result of this paper is a complete description of the response of the system, in terms of the firing-rate, to frequency variation. In particular, we prove that the firing-rate is maximal for a certain frequency which depends on the features of the stimulus (amplitude and duty cycle) as well as on the dynamical properties of the system. In addition, we provide detailed information on how to compute such frequency and the corresponding maximal value of the firing-rate.

This work is organized as follows.

In $\$ 2$ we describe the integrate-and-fire system, provide some definitions and state our results. In 3.1 we describe a bifurcation scenario established in our earlier work GKC13, which we use to prove our results. In $\$ 3.2$ we describe how the bifurcations in this parameter space change under frequency variation of the input. We also provide a precise statement of our results and their proofs. In 3.3 we present a result regarding the optimization of the firing-rate in terms of frequency of the input. Finally, in 4 we apply these results to an example, a linear integrate-and-fire neuron (LIF), to completely describe the firing-rate response under frequency variation. 


\section{The model, definitions and statement of re- sults}

In the context of neuronal modeling or hormone segregation one relies on excitable systems, which are able to exhibit certain responses given by large amplitude oscillations (spikes) as a response to certain stimulation. One of the most extended type of systems exhibiting this behavior are hybrid systems (a generalization of the so-called integrate-and-fire systems) which can be seen as approximation of slow/fast systems. That is, systems of the form

$$
\dot{x}=f(x)+I(t), x \in \mathbb{R},
$$

where $x$ represents an action potential or the output of the cell and $I(t)$ an external stimulation, which could be the output of another cell. Then, system 2.1) is submitted to the reset condition

$$
x=\theta \longrightarrow x=0,
$$

that is, the trajectories of system (2.1) are instantaneously reset to 0 whenever they reach the threshold given by $x=\theta$. Due to this instantaneous reset the solutions of the system exhibit discontinuities which emulate the spikes. In this work, we will consider $\theta$ a constant, although it is a common approach to add certain dynamics to this threshold in order to model more complex behaviours, as type III excitability MHR12.

As mentioned in the introduction, we will assume in this paper that the cell's input, $I(t)$, consists of a $T$-periodic square-wave function,

$$
I(t)= \begin{cases}A & \text { if } t \in(n T, n T+d T] \\ 0 & \text { if } t \in(n T+d T,(n+1) T]\end{cases}
$$

This is a well accepted, both in neuroscience and neuroendocrinology, to assume that inputs to excitable cells are given by functions of this form, as they occur in a pulsatile way. Other works consider rectified sinusoidals as inputs to neurons in the auditory brainstem MHR12.

The square wave function $I(t)$ will be characterized by three parameters: its amplitude $A$, its period $T$ and the duty cycle $d$, which is the duration of the pulse with respect to $T$.

As mentioned in $\$ 1$, the main goal of this work is to study the response of the system in terms of the firing-rate (number of spikes per unite time). In particular, we are interested on its optimization under the variation of parameters $A, d$ and $T$. However, we impose a constraint that the total amount of the released quantity be constant per stimulation period. We will refer to this as dose conservation, with the following biological question in mind: given a certain available quantity, how does it have to be released to the excitable cell in

order to obtain from it the highest firing-rate? Assuming that the experimental 
observation time, $\tau$, is large enough relative to the different periods of the signal $I(t), T$, the total amount of released quantity can be approximated by

$$
\int_{0}^{\tau} I(t) d t \sim Q \tau
$$

where $Q$ is the average value of $I$ over one period,

$$
Q:=\frac{1}{T} \int_{0}^{T} I(t) d t
$$

which we will call dose. Therefore, the cumulative dose released to the cell will be maintained as long as the dose $Q$ is conserved.

In order to add the dose conservation to our system, one has only to keep constant the product $A d$, which can be performed in different ways. In this work we will focus on two of them, the trivial one by keeping constant both $A$ and $d$ (width correction) and also the other one varying both $A$ and $d$ so that the total duration of the pulse, $\Delta=d T$, is constant (amplitude correction). In section 3 we will obtain theoretical results for the first case, which will be used also to study the behavior of the firing-rate under frequency variation for the second case in an example in 4.3 .

As mentioned above, the reset condition 2.2 introduces discontinuities to the solutions of the system. However, despite these discontinuities, the solutions of the non-autonomous system (2.1)- 2.2 are well defined. Let $\phi\left(t ; t_{0}, x_{0}\right)$ be the solution of system (2.1)-(2.2) fulfilling $\phi\left(t_{0} ; t_{0}, x_{0}\right)=x_{0}$. As usual in piecewisesmooth systems, the flow $\phi$ is obtained by properly matching the solutions for $0<t \bmod T \leq d T$ and $d T<t \bmod T \leq T$ combined with the reset condition 2.2. This makes the flow $\phi$ non-differentiable at $t \bmod T=d T$ and $t \bmod T=T$ and discontinuous at the spikes times, those at which the threshold is reached.

Remark 2.1. As we are interested in periodic orbits, although system 2.1)2.2) is non-autonomous, we will assume from now on that $t_{0}=0$, and we will write $\phi\left(t ; x_{0}\right)$. Note that if $t_{0} \neq 0$, the initial condition for a periodic orbit (fixed point of the stroboscopic map) may be different, although it still exists.

Let us assume that the system

$$
\dot{x}=f(x)
$$

satisfies the following conditions.

H.1 2.5 possesses an attracting equilibrium point

$$
0<\bar{x}<\theta,
$$

H.2 $f(x)$ is monotonic decreasing function in $[0, \theta]$ :

$$
f^{\prime}(x)<0,0 \leq x \leq \theta \text {. }
$$


As shown in GKC13, system (2.1)- 2.2) possesses attracting periodic orbits for almost all (except in a cantor set with zero measure) values of $A, \theta$ and $d$ as long as conditions H.1-H.2 are satisfied and $T$ is large or small enough. These periodic orbits may be continuous (subthreshold dynamics) or discontinuous (spiking dynamics). Let $\phi\left(t ; x_{0}\right)$, with $\phi\left(0 ; x_{0}\right)=x_{0}$, be an orbit of the nonautonomous system (2.1)- 2.2 . Then we consider

$$
r\left(x_{0}\right)=\lim _{\tau \rightarrow \infty} \frac{\#\left(\text { spikes performed by } \phi\left(t ; x_{0}\right) \text { for } t \in[0, \tau]\right)}{\tau},
$$

where \# means number of, if this limit exists. We then define the firing-rate.

Definition 2.1. If $r\left(x_{0}\right)$ does not depend on $x_{0}$ then we call it $r$, the firing-rate.

The firing-rate can be seen as the average number of spikes per unit time performed by the system along a periodic orbit.

Note that the firing-rate is well defined whenever there exists a unique attracting periodic orbit. However, it will in general depend on the system parameters $T$, $A$ and $d$.

Unlike in other approaches ( KHR81, CB99, CO00, COS01, CTW12, TB08), in order to study integrate-and-fire model $\sqrt{2.1})-\sqrt{2.2}$ our essential tool will be the stroboscopic map. Given an initial condition $x_{0}$, this map consists in flowing the system (2.1)- 2.2) for a time $T$, the period of the drive, and is the usual tool used when dealing with (smooth) periodic non-autonomous systems. In other words, it becomes

$$
\mathfrak{s}\left(x_{0}\right)=\phi\left(T ; x_{0}\right),
$$

where $\phi\left(t ; x_{0}\right)$ is the flow associated with (2.1)-(2.2). In the mentioned works, authors considered a Poincaré map from the threshold to itself (when spikes occur), added time as a variable and studied the times given by the spikes.

As it will be detailed below in $\$ 3.1$, the stroboscopic map will be piecewisedefined and discontinuous, and hence it is typically avoided in periodically forced hybrid systems, as one cannot apply classical results for regular smooth systems. These discontinuities of the map will not be given by the spikes performed by the trajectories of the system. On the contrary, the stroboscopic will undergo a discontinuity at those initial conditions for which the number of spikes performed by the trajectories for $t \in[0, T]$ changes (see Fig. 3.1). Despite these discontinuities, using results for non-smooth systems, the dynamics of the map is completely understood (see GKC13 for a discussion and references). This includes the rotation number, also called winding number, $\rho$, of all possible periodic orbits of the stroboscopic map, which will be of special interest in our work. The rotation number is usually associated with circle maps and, intuitively, measures the average rotation along trajectories when it does not depend on its initial condition. Under certain conditions, discontinuous piecewise-defined maps can be reduced to circle maps, and, when a periodic orbit exists, the rotation number becomes the ratio between the number of steps at the right of the discontinuity of the map along the periodic orbits to its period (see AGGK for more details). 
As shown in [KHR81] (see also [GKC13] and section \$3.1 below), the rotation number of the periodic orbits is well related with the number of spikes performed at each period of a periodic orbit of the stroboscopic map. A crucial quantity will be the average number of spikes by period of the stroboscopic map, which was named in KHR81 firing-number, $\eta$. This is given more precisely by the following definition.

Definition 2.2. Let $n$ be the total number of spikes performed by a p-periodic orbit of the stroboscopic map $\mathfrak{s}(x), n, p \in \mathbb{N}$; then we define the firing-number as

$$
\eta=\frac{n}{p},
$$

which is the average number of spikes per iteration of the stroboscopic map along a periodic orbit.

Remark 2.2. Then, assuming that the mentioned periodic orbit is attracting, the firing-rate can be obtained from the firing-number as

$$
r=\frac{\eta}{T} .
$$

As will be shown in $\$ 3.2$ (Corollary 3.1), depending on the value of the dose $Q$ defined in 2.4 the firing-rate will exhibit qualitatively different behaviors. This will bring us to consider a critical dose, which we define as follows.

Definition 2.3. The critical dose, $Q_{c}$, is the value of $A>0$ that places the equilibrium point, $\bar{x}$, of the system $\dot{x}=f(x)+A$ at the threshold; it is given by

$$
f(\theta)+Q_{c}=0 .
$$

Note that $Q_{c}$ is the minimal dose that permits the system (2.1)-(2.2) to exhibit spikes when it is driven constantly, $I(t)=Q_{c}\left(d=1\right.$ and $\left.A=Q_{c}\right)$.

Our goal is to study the qualitative behavior of the firing-rate under variation of the period of the input, $T$, for a chosen $Q$. We then prove the following results when $A>0$ and $d \in(0,1)$ are kept constant (width correction for dose conservation).

1. The firing-rate, obtained as the ratio of the firing-number $\eta$ to $T$, follows a devil's staircase with monotonically decreasing steps (see Figure 2.1) (except possibly in a compact set of values of $T$ ). This is a consequence of Propositions 3.1 and 3.2 (Corollary 3.2 .

2. The firing-rate for low frequency inputs fulfills

$$
\lim _{T \rightarrow \infty} r(T)=\frac{d}{\delta},
$$

where $\delta$ is the time needed by system $\dot{x}=f(x)+A$ to reach the threshold from $x=0$. This is Proposition 3.3 . 


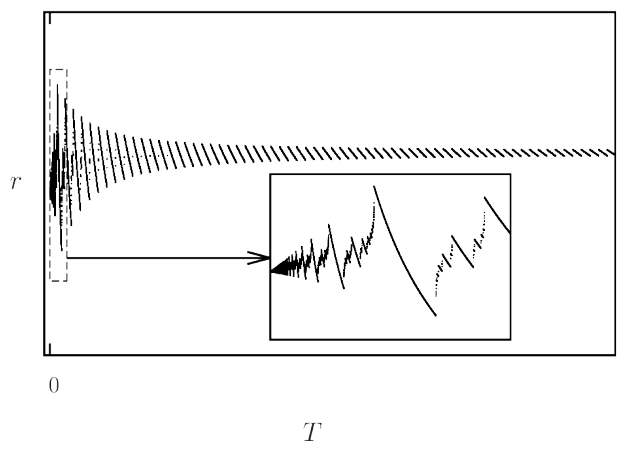

Figure 2.1: Typical response of the firing-rate upon frequency variation. It is given by a fractal structure of monotonically decreasing pieces, following a devil's staircase. The width of the "main pieces" stabilizes at $O(\delta / d)$, and each exhibits a local maximum and minimum. The firing-rate has limiting value $d / \delta$ when $T \rightarrow \infty$, and $1 / \hat{\delta}$ when $T \rightarrow 0$, and it exhibits a global maximum and minimum. See text (Section 2) for the definition of these parameters.

3. If $I(t)$ is such that $Q=A d<Q_{c}$, then the firing-rate becomes zero for large enough frequencies. This is also a consequence of Propositions 3.1 and 3.2 (Corollary 3.1).

4. If $Q>Q_{c}$, then

$$
\lim _{T \rightarrow 0} r(T)=\frac{1}{\hat{\delta}},
$$

where $\hat{\delta}>0$ is the time needed for the averaged system $\dot{x}=f(x)+A d$ to reach the threshold from $x=0$. This is Proposition 3.4 .

5. The firing-rate exhibits a global maximum and minimum in $T \in(0, \infty)$. Let $0<T_{1}<T_{2}$ such that $\eta(T)=1$ for $T \in\left[T_{1}, T_{2}\right]$. Then, if $T_{1}$ is large enough, the maximal firing rate occurs for $T=T_{1}$. The minimal one corresponds to the minimum between $0,1 / \hat{\delta}$ and $1 / T_{2}$. This is Proposition 3.5 and Remark 3.11.

\section{Bifurcation analysis}

\subsection{The two-dimensional parameter space}

In this section we provide a summary of the results shown in GKC13, see there for the details and proofs of what follows in this section.

As mentioned in $\$ 2$ due to the periodicity of $I(t)$, we will use the stroboscopic map 2.8), which is a discontinuous piecewise-smooth map, in order to 


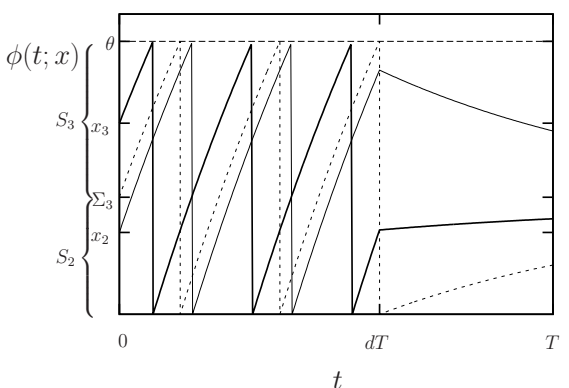

(a)

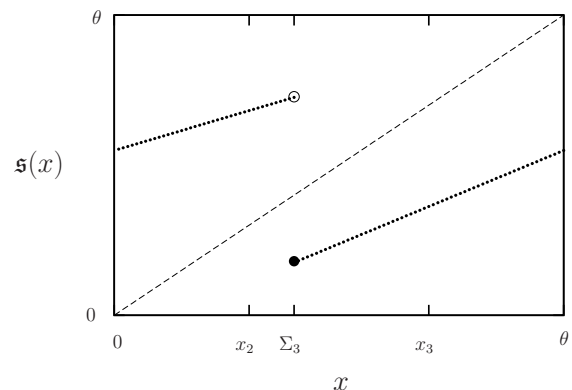

(b)

Figure 3.1: In (a) the trajectories of systems (2.1)-(2.2). Dashed line: trajectory with $\Sigma_{3}$ as initial condition. Solid thick line: trajectory with $x_{3}>\Sigma_{3}$ as initial condition, which spikes 3 times. Solid thin line: trajectory with $x_{2}<\Sigma_{3}$ as initial condition, which spikes 2 times. In (b) the stroboscopic map, with a discontinuity at $x=\Sigma_{3}$.

understand the dynamics of system (2.1)-(2.2). This map is a smooth map (as regular as $f(x)(2.1)$ ) in certain regions in the state space $[0, \theta]$ characterized by the number of spikes performed by $\phi$, the discontinuous flow associated with system (2.1)-2.2), when flowed for a time $T$. This is because, in these regions, the stroboscopic map becomes a composition of maps obtained by integrating system (2.1) and reseting from $x=\theta$ to $x=0$. Both types of intermediate maps are smooth. These regions in the state space are separated by boundaries of the form $x=\Sigma_{n}, \Sigma_{n}=\Sigma_{n}(A, T, d)$, where the stroboscopic map is discontinuous. At the right of $x=\Sigma_{n}$ the trajectories of (2.1)-(2.2) exhibit $n$ spikes when flowed for a time $T$, whereas at its left they exhibit $n-1$ spikes (see Figure 3.1 for $n=3$ ).

As the number of spikes can be arbitrarily large (for $A>0$ large enough), the state space $[0, \theta]$ is potentially divided in an infinite number of such regions. However, for fixed parameter values, the state space is split in at most two regions, $\left[0, \Sigma_{n}\right)$ and $\left[\Sigma_{n}, \theta\right)$, where the trajectories perform $n-1$ and $n$ spikes, respectively, when flowed during a time $T$. This comes from the fact that the initial condition that separates different sets of initial conditions leading to different number of spikes for $t \in[0, T]$ is unique, as it is the one spiking exactly at $t=d T$. We refer to GKC13 for further details.

The possible dynamics of the stroboscopic map, and hence of system (2.1)(2.2), is completely captured in the two-dimensional parameter space $d \times 1 / A$. Thus, by understanding the bifurcation structures in this parameter space one obtains a complete description of the fixed points, periodic orbits, their rotation 


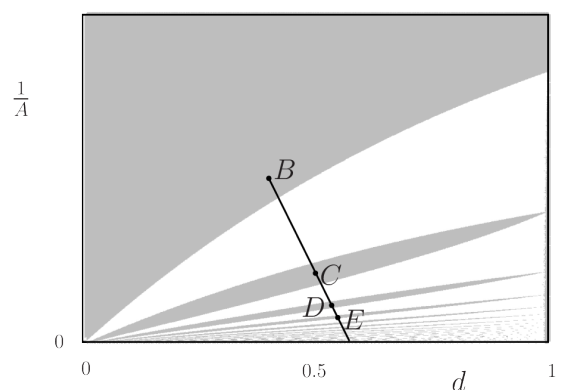

(a)

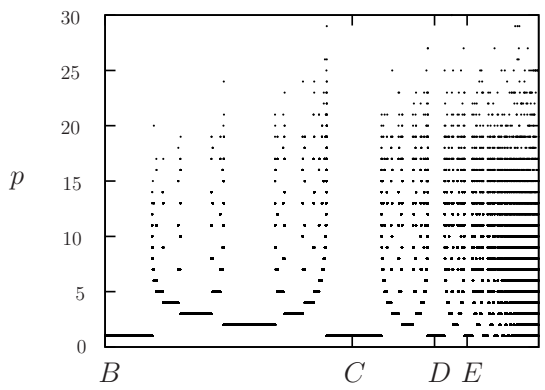

(b)

Figure 3.2: (a) Bifurcation scenario for system (2.1)-(2.2). In gray regions there exist $T$-periodic orbits. In the white regions, one finds higher periodic orbits following and adding structure (see text). In $B, C, D$ and $E$ one finds $T$-periodic orbits spiking $0,1,2$ and 3 times per period, respectively. (b) Periods of the periodic orbits found along the segment shown in (a).

numbers and their firing-rate.

Under the assumptions H.1-H.2, and if $T$ is small or large enough, the bifurcation scenario in the parameter space given by $d \times 1 / A$ for $T>0$ is equivalent to the one shown in Figure 3.2, which is described below and rigorously proven in GKC13. As suggested in Figure 3.2(a), there exists an infinite number of regions (in gray) accumulating to the horizontal axis for which only $T$-periodic orbits spiking $n$ times exist. These are fixed points, $\bar{x}_{n}$, of the stroboscopic map $\mathfrak{s}(x)(2.8)$. These regions in parameter space are ordered, in the clockwise direction, in such a way that these $T$-periodic orbits spike $0,1,2,3, \ldots$ times per period. The bifurcation curves that bound the gray regions are given by border collision bifurcation of the map. That is, the fixed points of the stroboscopic map $\bar{x}_{n}$ collide with one of the boundaries, $\bar{x}_{n}=\Sigma_{n}$ (Figures 3.3(a) and 3.4(a) for $n=3$ ) and $\bar{x}_{n}=\Sigma_{n+1}$ (Figures 3.3(d) and 3.4(d) for $n=3$ ), and no longer exist. This defines the upper and lower bifurcation curves, respectively, bounding each gray region as follows.

Definition 3.1. For $d \in(0,1)$, we define $A_{n}^{\mathcal{R}}(d)$ and $A_{n}^{\mathcal{L}}(d), n \geq 1$, the values of $A$ for which the fixed point $\bar{x}_{n}$ collides with the boundaries $\Sigma_{n}$ and $\Sigma_{n+1}$, respectively:

$$
\begin{gathered}
\lim _{A \rightarrow\left(A_{n}^{\mathcal{R}}\right)^{+}} \bar{x}_{n}=\Sigma_{n} \\
\lim _{A \rightarrow\left(A_{n}^{\mathcal{L}}\right)^{-}} \bar{x}_{n}=\Sigma_{n+1} .
\end{gathered}
$$


The fixed point $\bar{x}_{0} \in S_{0}$ undergoes only one border collision bifurcation, when it collides with $\Sigma_{1}$ from the left. This one occurs for $A=A_{0}(d)$,

$$
\lim _{A \rightarrow\left(A_{0}\right)^{-}} \bar{x}_{0}=\Sigma_{1} .
$$

Hence, a fixed point $\bar{x}_{n} \in[0, \theta]$ will exist if $A \in\left[A_{n}^{\mathcal{R}}, A_{n}^{\mathcal{L}}\right)$.

Remark 3.1. The values $A_{n}^{\mathcal{R}, \mathcal{L}}(d)$ depend also on $T$; we will explicitly specify this when convinient.

At the upper bifurcation curves $\left(A=A_{n}^{\mathcal{R}}(d)\right)$, the fixed points collide with a boundary from its right (Figures 3.3(a) and 3.4(a) , and hence will be associated to the $\mathcal{R}$ symbol. On the lower ones $\left(A=A_{n}^{\mathcal{L}}(d)\right)$ fixed points collide with another boundary from its left (see Figures 3.3(d) and 3.4(d) , and will have associated the symbol $\mathcal{L}$. Note that the stroboscopic map fulfills $\mathfrak{s}\left(\Sigma_{n}\right)=\mathfrak{s}\left(\Sigma_{n}^{+}\right)$, and hence the fixed points no longer exist at their left bifurcations whereas they still do for the right bifurcations (note the gray shown in Figures 3.3(d) and $3.4(\mathrm{~d})$. The first bifurcation defining the uppermost bifurcation curve, given by $\bar{x}_{0}=\Sigma_{1}\left(A=A_{0}(d)\right)$, is a bit different than the others, as it separates the parameter space $d \times 1 / A$ in two regions. In the lower side of the bifurcation curve, only spiking asymptotic dynamics are possible whereas on the upper side only a continuous $T$-periodic orbit exhibiting no spike can exist.

When entering the white regions, the map does no longer possess any fixed point. Instead, periodic orbits with arbitrarily high periods exist. These are shown in Figure 3.2(b) along the segment shown in Figure 3.2(a) As can be observed, they are organized by the period adding structure; that is, between two periodic orbits with periods $p$ and $q$, there exists another periodic orbit with period $p+q$.

As usual in piecewise-smooth dynamics, one can encode periodic orbits by introducing symbolic dynamics as follows. We assign the letters $\mathcal{L}$ and $\mathcal{R}$ depending on whether the corresponding periodic orbit steps on the left or on the right of the discontinuity. Then, the adding phenomenon is given by the concatenation of symbolic sequences; that is, between two regions in parameter space where the periodic orbits with symbolic sequences $\sigma$ and $\omega$ exist, there exists a region locating a periodic orbit with symbolic sequence $\sigma \omega$, whose period is the addition of the two previous ones. In Figure 3.5 we show the symbolic sequences of the periodic orbits found along the line shown in Figure $3.2(\mathrm{a})$ when crossing the white region between points $B$ and $C$, as well as their associated rotation numbers. These numbers are obtained by dividing the number of $\mathcal{R}$ 's contained in the symbolic sequence by its total length (the period of the periodic orbit). Note that the rotation numbers are organized by the so-called Farey tree associated with the period-adding phenomenon. Other authors [FG11] suggest that this should be given by a Stern-Brocot tree. However, in the context of the period adding, it is the Farey tree that needs to be considered, as it contains more precise information in the form of rotation numbers (see for example [GGT84, GIT84, and AGGK] for a recent survey.). As a consequence, the 


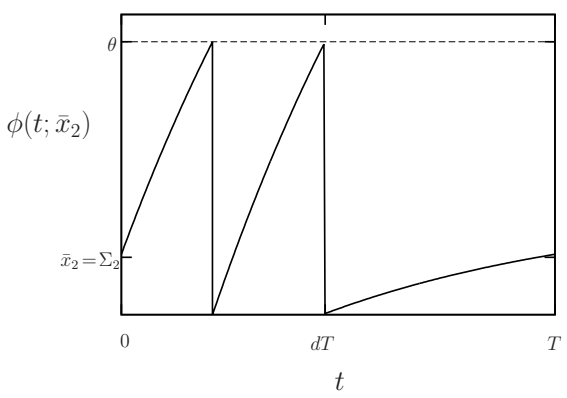

(a)

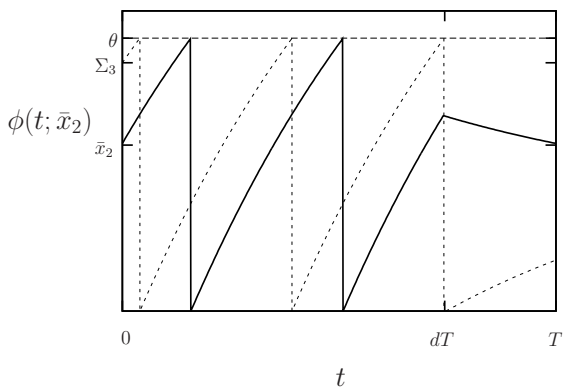

(c)

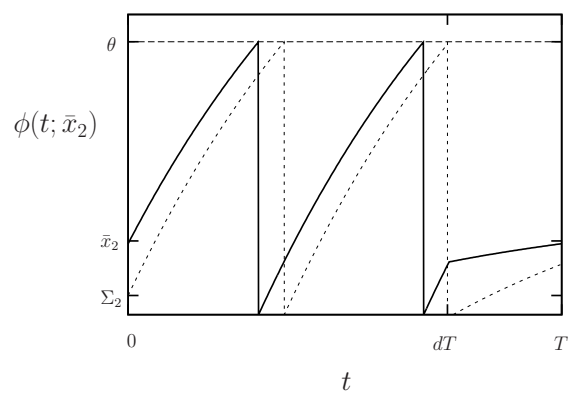

(b)

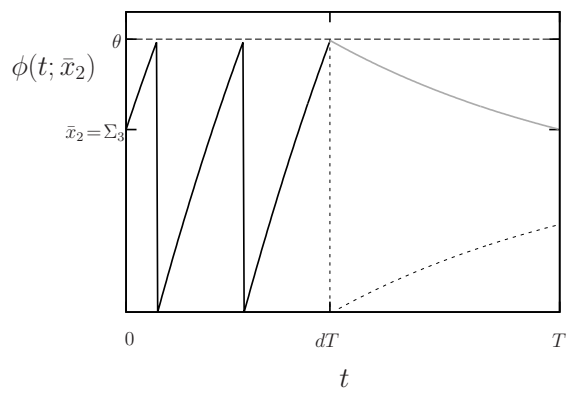

(d)

Figure 3.3: $T$-periodic orbit spiking twice per period (fixed point $\bar{x}_{2}$ of the stroboscopic map). It undergoes border collision bifurcations when it collides with the boundaries $\Sigma_{2}$ and $\Sigma_{3}$ (a) and (d), respectively. The periodic orbit shown in (d) is its limit when $\bar{x}_{2} \rightarrow \Sigma_{3}^{-}$; note that for $\bar{x}_{2}=\Sigma_{3}$ it should be reset to 0 at $t=d T$, this is why it is shown in gray. In (b) and (c), the trajectories of these boundaries are shown in dashed lines; note that they collide with the threshold at $t=d T$. Parameter values for panel (c) are the same as for point $D$ of Figure $3.2(\mathrm{a})$. The four figures are in one to one correspondence with the four figures of Figure 3.4, where the stroboscopic map is shown for the same parameter values. 


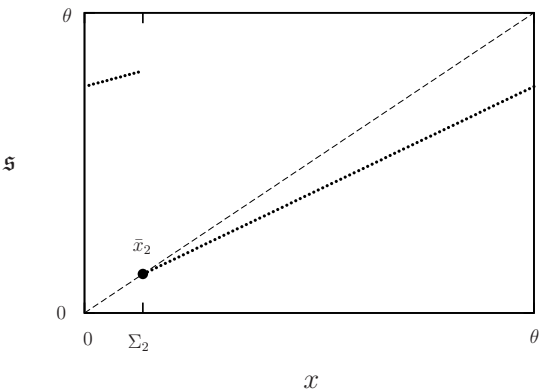

(a)

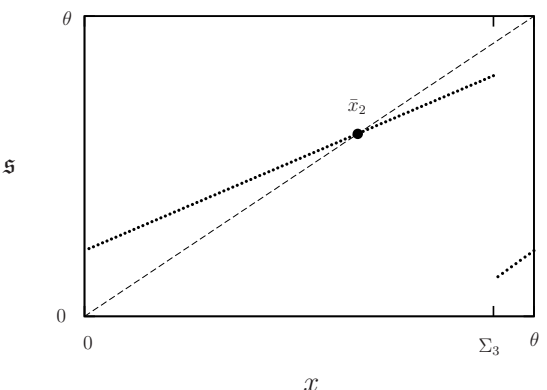

(c)

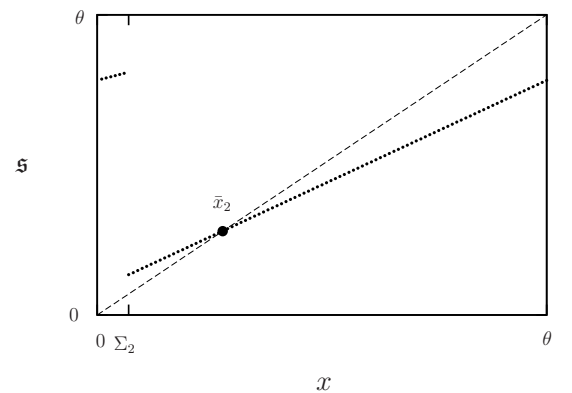

(b)

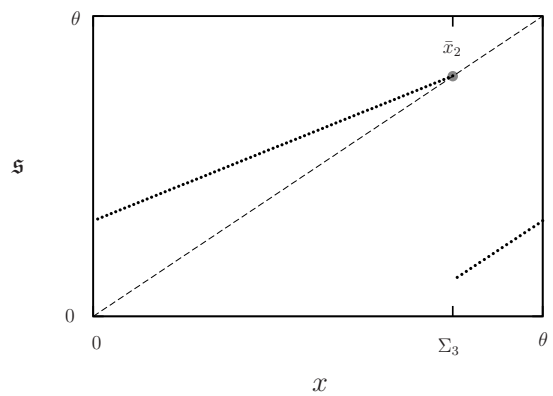

(d)

Figure 3.4: Stroboscopic map for the $T$-periodic orbits shown in Figure 3.3 In (a) and (d) the fixed point $\bar{x}_{2}$ undergoes border collision bifurcation when it collides with the boundaries $\Sigma_{2}$ from the right and $\Sigma_{3}$ from the left, respectively. Note that, in (d) the fixed point is shown in gray to emphasize that the map takes indeed the value on the right for $x=\Sigma_{3}$. In (b)-(c) the boundary $\Sigma_{2}$ disappears and a new boundary $\Sigma_{3}$ appears while the fixed point $\bar{x}_{2}$ remains. 


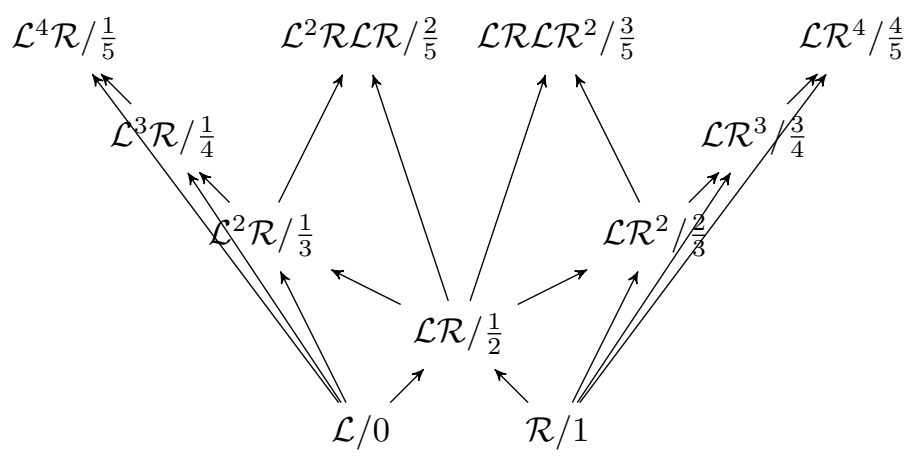

Figure 3.5: Symbolic sequences and rotation numbers forming the so-called Farey tree structure for the period adding. The symbol $\mathcal{L}$ corresponds to a step on the left of the discontinuity, $\mathcal{R}$ to a step on its right.

rotation number follows a devil's staircase from 0 to 1 . This is a monotonically increasing function which is constant almost everywhere, except in a Cantor set of zero measure.

Immediately after crossing a white region and entering a gray one where another $T$-periodic orbit exists (fixed point of the stroboscopic map), the rotation number equals 1 for a while until it suddenly jumps to 0 again. This is due to the following reason.

When varying parameters along the line shown in $3.2(\mathrm{a})$ inside the gray regions, a new discontinuity $\Sigma_{n+1}$ enters $[0, \theta]\left(\theta=\Sigma_{n+1}\right)$ and $\Sigma_{n}$ no longer exists (because of the uniqueness of the discontinuity mentioned above), while the periodic orbit spiking $n$ times still exists and hence is not subject to any bifurcation (see Figures 3.4(b) 3.4(c) for $n=2$ ). At this moment, however, the rotation number associated with this periodic orbit jumps from 1 to 0 , and the state space is now split in two pieces, $\left[0, \Sigma_{n+1}\right)$ and $\left[\Sigma_{n+1}, \theta\right)$ where the system spikes $n$ and $n+1$ times, respectively. This comes from the fact that, when a new discontinuity appears, what was on the right of the previous discontinuity $\Sigma_{n}$ becomes on the left of the new one $\Sigma_{n+1}$; hence, the stroboscopic map can be reduced to a new circle map which rotates on the opposite direction.

Remark 3.2. The symbols $\mathcal{L}$ and $\mathcal{R}$ in the symbolic sequences of the periodic orbits located in the white regions correspond to $n$ and $n+1$ spikes in a T-time interval, respectively.

Remark 3.3. Following GIT84, one can relate the rotation numbers to the symbolic dynamics associated with the periodic orbits that appear along the line shown in Figure 3.2(a), by dividing the number of $\mathcal{R}$ 's that appear in the symbolic sequence by the period of the periodic orbit. Hence, taking into account Remark 3.2, between $B$ and $C$ in Figure 3.2(a) the rotation number equals the firing-number $\eta$. 
Remark 3.4. Beyond point $C$ in the line shown in fig. 3.2( $a$ ), $\eta$ varies along the line as the rotation number but without the jumping from 1 to 0 . Hence, this quantity follows a devil's staircase from 0 to $\infty$ when parameters are varied along such a line.

Remark 3.5. In the conditions mentioned at the beginning of this section, in addition to H.1-H.2 it was also required that $T$ be large or small enough. This is needed in order to ensure that the stroboscopic map $\mathfrak{s}$ is contractive in all its domain, which is a necessary condition for the occurrence of the period adding. It is possible, for certain values of $T$, that, when $A$ is not sufficiently large, the stroboscopic map $\mathfrak{s}$ be expanding in the domain $\left[\Sigma_{n}, \theta\right]$. When this occurs, the rotation number may no longer follow a devil's staircase but a continuous increasing function, the existence of a periodic orbit may not be unique and it can unstable. See [GKC13] for more details.

\subsection{Bifurcation scenario upon frequency variation}

We now focus on how the bifurcation scenario described in $\$ 3.1$ and schematically shown in Figure 3.2 varies with $T$.

As proven in GKC13] this bifurcation scenario does not qualitatively depend on $T$ and, hence, no other bifurcations are introduced nor subtracted under variation of $T$ as long as contractivness of the stroboscopic map $\mathfrak{s}$ is kept (see Remark 3.5). However, the shape of the bifurcation curves varies, as the next two propositions show (see Figures 4.1 and 4.2 for graphical support through an example). Proposition 3.1 tells us that the bifurcation curves accumulate to the horizontal line $1 / A=1 / Q_{c}$ when $T \rightarrow \infty$ (labeled in all paths of Figures 4.1 and 4.2 . Proposition 3.2 tells us that all bifurcation curves accumulate to horizontal axis, except for the one given by $A_{0}(d)$, which accumulates to the straight line $1 / A=d / Q_{c}$ when $T \rightarrow 0$ (see Figure 4.1(a)).

Proposition 3.1. Let $d \in(0,1)$ and consider the values given in definition 3.1. $A_{n}^{\mathcal{R}, \mathcal{L}}=A_{n}^{\mathcal{R}, \mathcal{L}}(d, T)$ and $A_{0}=A_{0}(d, T)$, for which the fixed points $\bar{x}_{n}$ undergo border collision bifurcations. Then,

$$
\lim _{T \rightarrow \infty} A_{0}(d, T)=\lim _{T \rightarrow \infty} A_{n}^{\mathcal{R}}(d, T)=\lim _{T \rightarrow \infty} A_{n}^{\mathcal{L}}(d, T)=Q_{c},
$$

where $Q_{c}$ is the critical dose defined in 2.11.

Proof. Let $\varphi(t ; x ; A)$ be the flow associated with system $\dot{x}=f(x)+A$, and let $\bar{x}_{n}=\bar{x}_{n}(d, T, A)$ be the initial condition $\left(t_{0}=0\right)$ for a $T$-periodic orbit spiking $n$ times (fixed point of the stroboscopic map $\mathfrak{s}(x)$ ). As shown in Figure 3.6 . (see GKC13 for more details), the border collision bifurcations that the $T$ periodic orbit spiking $n$ times undergo at $A=A_{n}^{\mathcal{R}}(d, T)$ (fig. 3.6(a) and $A=A_{n}^{\mathcal{L}}$ 
(fig. $3.6(\mathrm{~b})$ are characterized by the equations

$$
\begin{aligned}
\varphi\left(t_{n} ; \bar{x}_{n} ; A_{n}^{\mathcal{R}}\right) & =\theta \\
\varphi\left(\delta ; 0 ; A_{n}^{\mathcal{R}}\right) & =\theta \\
\varphi(T-d T ; 0 ; 0) & =\bar{x}_{n} \\
t_{n}+(n-1) \delta & =d T
\end{aligned}
$$

and

$$
\begin{aligned}
\varphi\left(t_{n}^{\prime} ; \bar{x}_{n} ; A_{n}^{\mathcal{L}}\right) & =\theta \\
\varphi\left(\delta^{\prime} ; 0 ; A_{n}^{\mathcal{L}}\right) & =\theta \\
\varphi(T-d T ; \theta ; 0) & =\bar{x}_{n} \\
t_{n}^{\prime}+(n-1) \delta^{\prime} & =d T,
\end{aligned}
$$

respectively. As $n$ is fixed, when $T \rightarrow \infty$ also $\delta \rightarrow \infty$. Hence, from equations (3.1) and (3.2) we get that the values $A=A_{n}^{\mathcal{R}}$ and $A=A_{n}^{\mathcal{L}}(d, T)$ are such that system $\dot{x}=f(x)+A$ possesses an attracting critical point at $x=\theta$. From equation 2.11) we get that the limiting values are $A_{n}^{\mathcal{R}}=A_{n}^{\mathcal{L}}=Q_{c}$.

Arguing similarly and using that the bifurcation condition for $A_{0}(d, T)$ is equivalent to 3.2 , one gets that $A_{0} \rightarrow Q_{c}$.

Proposition 3.2. Let $d \in(0,1)$ and consider the bifurcation values of the fixed points $\bar{x}_{n} \in S_{n}, A_{0}(d, T)$ and $A_{n}^{\mathcal{R}, \mathcal{L}}(d, T)(n>1)$ as in definition 3.1. Then they fulfill

i)

$$
\lim _{T \rightarrow 0} A_{0}(d, T)=\frac{Q_{c}}{d},
$$

where $Q_{c}$ is the critical dose defined in 2.11).

ii)

$$
\lim _{T \rightarrow 0} A_{n}^{\mathcal{R}, \mathcal{L}}(d, T)=\infty, n>1 .
$$

Proof. Let $\varphi(t ; x ; A)$ be the flow associated with the system $\dot{x}=f(x)+A$. The fact that $f(x)$ is a monotonously decreasing function with a simple zero (the equilibrium point $\bar{x}$ in H.1) ensures that the bifurcation suffered by the non-spiking $T$-periodic orbit will be given when

$$
\left\{\begin{array}{l}
\varphi\left(d T ; \bar{x}_{0} ; A_{0}\right)=\theta \\
\varphi(T-d T ; \theta ; 0)=\bar{x}_{0},
\end{array}\right.
$$

where $\bar{x}_{0}$ is the initial condition for such periodic orbit for $t=0$. We want to solve these equations for $A_{0}$ and $\bar{x}_{0}$ for a fixed $d \in(0,1)$ and $T>0$, and see 


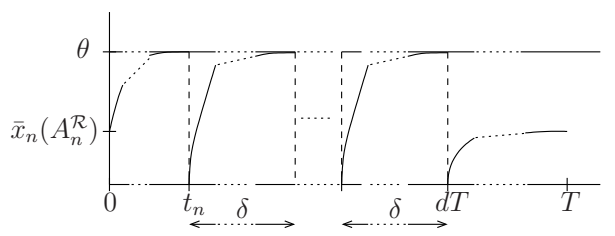

(a)

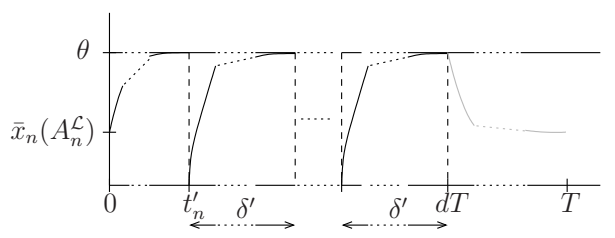

(b)

Figure 3.6: Bifurcations of the $T$-periodic spiking $n$ times for large values of $T$. (a) and (b) smallest and largest values of $A\left(A_{n}^{\mathcal{R}}\right.$ and $A_{n}^{\mathcal{L}}$, respectively) for which the $T$-periodic orbit exists. This corresponds to the border collision bifurcations given by $\bar{x}_{n}\left(A_{n}^{\mathcal{R}}\right)=\Sigma_{n}$ and $\bar{x}_{n}\left(\left(A_{n}^{\mathcal{L}}\right)^{-}\right)=\Sigma_{n+1}$, respectively. The gray color in (b) reflects the fact that such orbit does no longer exist for $A=A_{n}^{\mathcal{L}}$, as it must be reset to 0 at $t=d T$. The orbit shown is the limiting periodic orbit for $A \rightarrow A_{n}^{\mathcal{L}}$.

how this solution behaves when $T \rightarrow 0$.

For $T \rightarrow 0$ we can approximate the flow by a linear one and obtain

$$
\begin{aligned}
& \varphi\left(d T ; x ; A_{0}\right)=x+\left(f(x)+A_{0}\right) d T+O\left(T^{2}\right) \\
& \varphi(T-d T ; \theta ; 0)=\theta+f(\theta)(1-d) T+O\left(T^{2}\right) .
\end{aligned}
$$

Hence, for $T \rightarrow 0$, the bifurcation condition 3.4 becomes equivalent to the system

$$
\left\{\begin{array}{c}
\bar{x}_{0}+\left(f\left(\bar{x}_{0}\right)+A_{0}\right) d T=\theta \\
\theta+f(\theta)(1-d) T=\bar{x}_{0},
\end{array}\right.
$$

which we can use to obtain the explicit expression

$$
A_{0}(d, T)=-\frac{f(\theta)}{d}+f(\theta)-f(\theta+f(\theta)(1-d) T)+O\left(T^{2}\right) .
$$

Recalling the definition of the critical dose given in Eq. (2.11), $Q_{c}=-f(\theta)$, we obtain

$$
\lim _{T \rightarrow 0} A_{0}(d, T)=\frac{Q_{c}}{d},
$$

which proves i).

To see that all other bifurcation curves accumulate at the horizontal line $1 / A=$ 
0 , as stated in $i i)$, we just use the fact that the periodic orbits involved in these bifurcations perform at least one spike for $t \in[0, d T]$. Hence, when $T \rightarrow 0$, we necessary have that $A \rightarrow \infty$ in order to keep these spikes.

Remark 3.6. Note that the fact that the border collision bifurcation curves defined by $A_{n}^{\mathcal{R}, \mathcal{L}}$ collapse to the horizontal axis for $T \rightarrow 0$ implies that all other border collision bifurcation curves separating regions of existence of periodic orbits with higher periods also collapse to the horizontal axis, as they are located in between.

The next result tells us that all bifurcation curves vary monotonically with $T$.

Lemma 3.1. For a fixed $d \in(0,1)$ let $A^{\mathcal{R}}(d, T)$ and $A^{\mathcal{L}}(d, T)$ be the values for which a periodic orbit undergoes a border collision bifurcation. Then they are monotonic functions of $T$.

Proof. We prove the result for the bifurcations undergone by fixed points of the stroboscopic map $\left(A_{n}^{\mathcal{L}}(d, T)\right.$ and $\left.A_{n}^{\mathcal{R}}(d, T)\right)$. Proceeding similarly one obtains the analogous result for periodic orbits.

Assume that $\bar{x}_{n}$ is a fixed point of the stroboscopic map $\mathfrak{s}(x)$ leading to a $T$-periodic orbit exhibiting $n$ spikes per period. Such a fixed point undergoes a left bifurcation for $A=A_{n}^{\mathcal{L}}$ when the following equations are satisfied (see fig. $3.3(\mathrm{~d})$

$$
\begin{aligned}
& \int_{\bar{x}_{n}}^{\theta} \frac{d x}{f(x)+A_{n}^{\mathcal{L}}}+n \int_{0}^{\theta} \frac{d x}{f(x)+A_{n}^{\mathcal{L}}}=a T \\
& \int_{\theta}^{\bar{x}_{n}} \frac{d x}{f(x)}=(1-a) T,
\end{aligned}
$$

where we have renamed the duty cycle $d$ by $a$ to avoid the confusion with the notation used for derivatives and differentials. Differentiating the previous equations with respect to $T$ we get

$$
\begin{aligned}
a & =-\frac{d A_{n}^{\mathcal{L}}}{d T}\left(\int_{\bar{x}_{n}}^{\theta} \frac{d x}{\left(f(x)+A_{n}^{\mathcal{L}}\right)^{2}}+n \int_{0}^{\theta} \frac{d x}{\left(f(x)+A_{n}^{\mathcal{L}}\right)^{2}}\right)-\frac{1}{f\left(\bar{x}_{n}\right)+A_{n}^{\mathcal{L}}} \frac{d \bar{x}_{n}}{d T} \\
\frac{d \bar{x}_{n}}{d T} & =(1-a) f\left(\bar{x}_{n}\right) .
\end{aligned}
$$

We want to see that $d A_{n}^{\mathcal{L}} / d T<0$. Combining these last equations we get

$$
-\left(\int_{\bar{x}_{n}}^{\theta} \frac{d x}{\left(f(x)+A_{n}^{\mathcal{L}}\right)^{2}}+n \int_{0}^{\theta} \frac{d x}{\left(f(x)+A_{n}^{\mathcal{L}}\right)^{2}}\right) \frac{d A_{n}^{\mathcal{L}}}{d T}=a+(1-a) \frac{f\left(\bar{x}_{n}\right)}{f\left(\bar{x}_{n}\right)+A_{n}^{\mathcal{L}}} .
$$

We note that

- the coefficient of $d A_{n}^{\mathcal{L}} / d T$ in the left hand side is negative 
- $f\left(\bar{x}_{n}\right)\left(f\left(\bar{x}_{n}\right)+A_{n}^{\mathcal{L}}\right)<0$.

Hence, to prove the result we need to show that

$$
a+(1-a) \frac{f\left(\bar{x}_{n}\right)}{f\left(\bar{x}_{n}\right)+A_{n}^{\mathcal{L}}}>0 .
$$

We know that if $T \rightarrow \infty$ then $f\left(\bar{x}_{n}\right) \rightarrow 0$ and $A_{n}^{\mathcal{L}} \rightarrow Q_{c}$ (Proposition 3.1). Hence (3.5) holds for large $T$. We will prove that $a+(1-a) f\left(\bar{x}_{n}\right) /\left(f\left(\bar{x}_{n}\right)+A\right)=0$ is not possible. First note that this equation is equivalent to $\bar{x}_{n}=f^{-1}(-a A)$. Hence we consider the following system of three equations:

$$
\begin{aligned}
& \int_{\bar{x}_{n}}^{\theta} \frac{d x}{f(x)+A_{n}^{\mathcal{L}}}+n \int_{0}^{\theta} \frac{d x}{f(x)+A_{n}^{\mathcal{L}}}=a T \\
& \int_{\theta}^{\bar{x}_{n}} \frac{d x}{f(x)}=(1-a) T \\
& \bar{x}_{n}=f^{-1}\left(-a A_{n}^{\mathcal{L}}\right) .
\end{aligned}
$$

and we show that the three equations in (3.6) cannot be simultaneously satisfied. Eliminating the variables we get

$$
\int_{f^{-1}\left(-a A_{n}^{\mathcal{L}}\right)}^{\theta}\left(\frac{a}{f(x)}+\frac{1-a}{f(x)+A_{n}^{\mathcal{L}}}\right) d x+n \int_{0}^{\theta} \frac{(1-a)}{f(x)+A_{n}^{\mathcal{L}}} d x=0 .
$$

On one hand, $A_{n}^{\mathcal{L}}$ is large enough to make the system spike, and hence $f(\theta)+$ $A_{n}^{\mathcal{L}}>0$. On the other hand, as $\bar{x}_{n}<\theta$, from the last equation of Eq. (3.6) we get that $-a A_{n}^{\mathcal{L}}<f(\theta)$. Therefore, we know that $0<-f(\theta) \leq A_{n}^{\mathcal{L}}<-f(\theta) / a$. We define the function

$$
\psi(A)=\int_{f^{-1}(-a A)}^{\theta}\left(\frac{a}{f(x)}+\frac{1-a}{f(x)+A}\right) d x+n \int_{0}^{\theta} \frac{(1-a)}{f(x)+A} d x .
$$

Clearly $\psi(-f(\theta) / a)>0$. We compute

$$
\begin{aligned}
\psi^{\prime}(A) & =\frac{a}{f^{\prime}\left(f^{-1}(-a A)\right)}\left(-\frac{1}{A}+\frac{1}{A}\right)-\int_{f^{-1}(-a A)}^{\theta} \frac{1-a}{(f(x)+A)^{2}} d x \\
& -n \int_{0}^{\theta} \frac{1-a}{(f(x)+A)^{2}} d x \\
& =-\int_{f^{-1}(-a A)}^{\theta} \frac{1-a}{(f(x)+A)^{2}} d x-n \int_{0}^{\theta} \frac{1-a}{(f(x)+A)^{2}} d x<0 .
\end{aligned}
$$

Hence $\psi$ is strictly monotonic so it cannot have a 0 in $[-f(\theta),-f(\theta) / a)$.

We now show that the same holds for the right bifurcations of the fixed points (see fig. 3.3(a)]; that is, $d A_{n}^{\mathcal{R}} / d T<0$. 
In this case, the equations that determine such bifurcation become

$$
\begin{aligned}
& \int_{\bar{x}_{n}}^{\theta} \frac{d x}{f(x)+A_{n}^{\mathcal{R}}}+n \int_{0}^{\theta} \frac{d x}{f(x)+A_{n}^{\mathcal{R}}}=a T \\
& \int_{0}^{\bar{x}_{n}} \frac{d x}{f(x)}=(1-a) T,
\end{aligned}
$$

Unlike in the previous case, $\bar{x}_{n}$ increases to $\bar{x}$ (the equilibrium point assumed in H.1) when $T$ is increased. This leads to a decrease of the time of the first spike (value of the first integral). Hence, one necessary needs to decrease $A_{n}^{\mathcal{R}}$ in order to keep these equations satisfied.

We will now use Propositions 3.1 and 3.2 to derive information about the behavior of the firing-rate for large and small periods, Propositions 3.3 and 3.4 . respectively. First we present the next corollary of Propositions 3.1, 3.2 and Lemma 3.1. It provides a partition of the parameter space in three different regions regarding spiking properties for different values of $T$.

Corollary 3.1. The parameter space $d \times 1 / A$ is divided in three main regions with the following properties

- Non-spiking region,

$$
\left\{(d, 1 / A) \in \mathbb{R}^{2} \mid d \in(0,1), A<Q_{c}\right\},
$$

for which the corresponding periodic orbit does not contain any spike for any $d \in(0,1)$ and $T>0$.

- Permanent-spiking region,

$$
\left\{(d, 1 / A) \in \mathbb{R}^{2} \mid d \in(0,1), A>Q_{c} / d\right\},
$$

for which, the existing periodic orbit contains spikes for all $T>0$.

- Conditional-spiking region,

$$
\left\{(d, 1 / A) \in \mathbb{R}^{2} \mid d \in(0,1), Q_{c}<A<Q_{c} / d\right\},
$$

for which there exists $T_{0}>0$ such that the corresponding periodic orbit contains spikes if $T>T_{0}$ and does not if $T<T_{0}$.

Remark 3.7. The spiking-region is formed by the union of the conditional and permanent-spiking regions.

Corollary 3.2. If $(d, 1 / A)$ belongs to the spiking-region, then, for those values of $T$ for which $\mathfrak{s}$ is contracting in $[0, \theta]$, the firing-rate $r(T)$ follows a devil's staircase with monotonically decreasing steps. For the values of $T$ for which $\mathfrak{s}$ loses contractiveness $r(T)$ is a monotonically increasing function. 
Proof. It follows from Propositions 3.1, 3.2 and Lemma 3.1 that, as long as $T$ is such that $\mathfrak{s}$ is contracting (see Remark 3.5 , then the bifurcation lines defining the steps of the devil's staircase move up monotonically as $T$ is increased. Hence, if we fix $(d, 1 / A)$ in the spiking region, then as $T$ increases all the bifurcation curves pass through the point $(d, 1 / A)$ and the bifurcation diagram is the same as when $d$ and $T$ are fixed and $A$ is varied (i.e. when varying parameters along the line shown in fig. $3.2(\mathrm{a})$ for a fixed $T$ ).

Recall that the the rotation number follows a devil's staircase which is constant along the steps. Then, using Remarks 3.3 and 3.4 and formula 2.10 we conclude that $r(T)$ follows a devil's staircase which is monotonically decreasing along the steps.

If eventually $\mathfrak{s}$ becomes expanding in $\left[\Sigma_{n}, \theta\right]$, then the rotation number does not follow a devil's staircase but a monotonically increasing continuous function (see GKC13, AGGK for more details).

From the previous results we get the next corollary providing the behaviour of the firing number $\eta$ for large and small values of $T$.

Corollary 3.3. In the spiking region the firing number defined in 2.2 (average number of spikes per iteration of the stroboscopic map) satisfies

$$
\begin{aligned}
& \lim _{T \rightarrow 0} \eta(T)=0 \\
& \lim _{T \rightarrow \infty} \eta(T)=\infty .
\end{aligned}
$$

Morover, in the conditional spiking region $\eta(T)=0$ for $0<T<T_{0}=T_{0}(A, d)$.

Note that the relation between the firing number and firing-rate given by equation 2.10 implies that their asymptotic behavior is not necessarily the same when $T \rightarrow \infty$ or $T \rightarrow 0$. We will now use the results obtained so far in this section to characterize the limits of the firing-rate as $T \rightarrow \infty$ and as $T \rightarrow 0$. The following result describes the limit of $r(T)$ as $T \rightarrow \infty$ for points in the spiking region.

Proposition 3.3. Let $(d, 1 / A)$ belong to the spiking-region $(d \in(0,1)$ and $A>$ $\left.Q_{c}\right)$ and let $\varphi(t ; x ; A)$ be the flow associated with $\dot{x}=f(x)+A$. Let $\delta>0$ be the smallest value such that

$$
\varphi(\delta ; 0 ; A)=\theta
$$

Then, the firing-rate satisfies

$$
\lim _{T \rightarrow \infty} r(T)=\frac{d}{\delta}
$$

Proof. We will show that the devil's staircase followed by the firing number $\eta(T)$ converges to a common discontinuous staircase whose each step has length $\delta / d$. These steps have integer values, $n$, and correspond to $T$-periodic orbits spiking $n$ times. More precisely, we prove that

$$
\lim _{T \rightarrow \infty} \eta(T)-E\left(\frac{\delta T}{d}\right) \rightarrow 0,
$$


where $E(x)$ is the integer value of $x$. The result follows from (3.10) and from the definition of firing-rate.

To prove (3.10) we focus on a $T$-periodic orbit with $n$ spikes per period. Let $\bar{x}_{n}(T)$ be the initial condition $\left(t_{0}=0\right)$ for such an orbit (fixed point of the stroboscopic map). This fulfills

$$
\begin{aligned}
\varphi\left(t_{n} ; \bar{x}_{n}(T) ; A\right) & =\theta \\
\varphi\left(d T-(n-1) \delta-t_{1} ; 0 ; A\right) & =x^{\prime} \\
\varphi\left(T-d T ; x^{\prime} ; 0\right) & =\bar{x}_{n}(T),
\end{aligned}
$$

for some $0<t_{n}<\delta$. The last equation tells us that

$$
\lim _{T \rightarrow \infty} \bar{x}_{n}(T)=\bar{x},
$$

where $\bar{x}$ is the equilibrium point 2.6 associated with system $\dot{x}=f(x)$ given by assumptions H.1-H.2.

At the same time, this tells us that the stroboscopic map converges to a constant function equal to $\bar{x}$. Recalling that the discontinuities of the stroboscopic map occur at $x=\Sigma_{i}$, the gaps at these discontinuities tend to zero,

$$
\lim _{T \rightarrow \infty} \mathfrak{s}\left(\Sigma_{n}^{-}\right)=\lim _{T \rightarrow \infty} \mathfrak{s}\left(\Sigma_{n}^{+}\right)=\bar{x} .
$$

Hence, when $T \rightarrow \infty$ there is no space for periodic orbits with higher periods. In other words, let $T_{n}^{\mathcal{R}}$ and $T_{n}^{\mathcal{L}}$ be the values of $T$ for which a $T$-periodic orbit spiking $n$ times appears and disappears through border collisions bifurcations, respectively (Figures 3.7(a) and 3.7(c), respectively). Then we have that

$$
\lim _{T \rightarrow \infty} T_{n+1}^{\mathcal{R}}-T_{n}^{\mathcal{L}}=0,
$$

and the devil's staircase converges to be a common staircase. Its steps are given by integer values $n$, as they correspond to the firing-number associated with $T$-periodic orbits spiking $n$ times.

We now estimate the length of these steps when $T \rightarrow \infty$. From equation (3.11) we get that, as $T \rightarrow \infty, t_{1}$ converges to the solution of the equation

$$
\varphi\left(t_{1} ; \bar{x} ; A\right)=\theta .
$$

As, for a fixed value of $A$ and $d$ in the spiking region, the number of spikes performed by a $T$-periodic orbit tends to infinity as $T \rightarrow \infty$ (Proposition 3.1), the interval of time where the spikes occur is of order $n \delta$. Hence, taking into account the characteristics of the $T$-periodic orbit at its bifurcation (see Figures 3.7(c), 3.7(a) and GKC13), we get

$$
\begin{aligned}
d T_{n}^{\mathcal{R}} & \sim n \delta \\
d T_{n}^{\mathcal{L}} & \sim(n+1) \delta,
\end{aligned}
$$




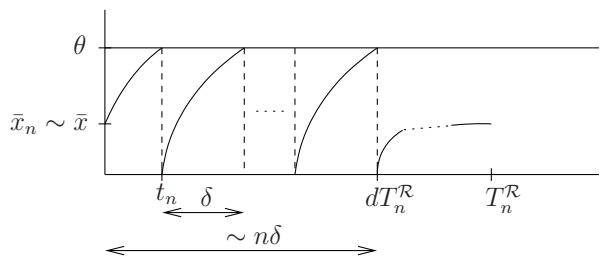

(a)

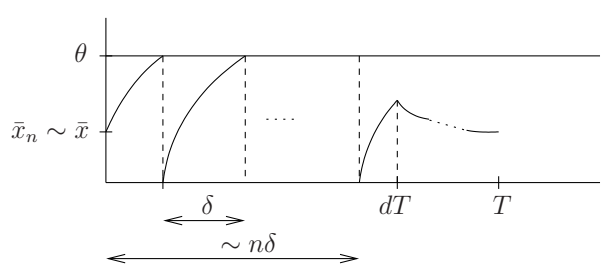

(b)

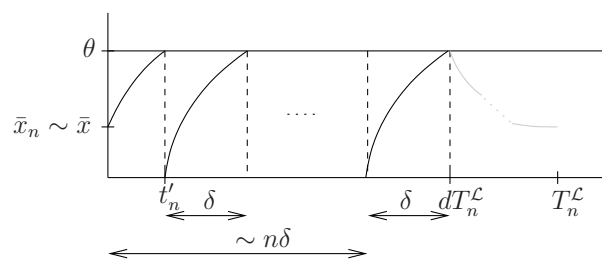

(c)

Figure 3.7: $T$-periodic orbit spiking $n$ times at its bifurcations for large values of $T$. The periodic orbit appears (a) and disappears (c) through border collision bifurcations at $T=T_{n}^{\mathcal{R}}$ and $T=T_{n}^{\mathcal{L}}$, respectively. In (b) a $T$-periodic orbit for $T \in\left(T_{n}^{\mathcal{R}}, T_{n}^{\mathcal{L}}\right)$. The gray color emphasizes the fact that the periodic orbit does not exist for $T=T_{n}^{\mathcal{L}}$ as it should be reset to 0 when the threshold is reached. The orbit shown is the limiting periodic orbit when $T \rightarrow\left(T_{n}^{\mathcal{L}}\right)^{-}$. In all three cases the fixed point $\bar{x}_{n}$ approaches $\bar{x}$, the critical point of system 2.5 as $T \rightarrow \infty$. 
and thus

$$
T_{n}^{\mathcal{L}}-T_{n}^{\mathcal{R}} \sim \frac{\delta}{d}
$$

which is the length of the step with integer value.

We end this section with a result which describes the behavior of $r(T)$ as $T \rightarrow 0$.

Proposition 3.4. Let $(d, 1 / A)$ belong to the spiking-region, and let

$$
\dot{x}=f(x)+A d
$$

be the averaged version of system 2.1. Let $\hat{\varphi}(t ; x)$ be its associated flow and let $\hat{\delta}>0$ be the smallest number such that

$$
\hat{\varphi}(\hat{\delta} ; 0)=\theta .
$$

Then,

- if $(d, 1 / A)$ belongs to the conditional-spiking region $\left(A d<Q_{c}\right)$ then $r(T)=$ 0 if $T<T_{0}$, where $T_{0}$ is given in Corollary 3.1 .

- if $(d, 1 / A)$ belongs to the permanent-spiking region $\left(A d>Q_{c}\right)$, then

$$
\lim _{T \rightarrow 0} r(T)=\frac{1}{\hat{\delta}}
$$

Proof. For the first case we use Corollary 3.1. from which we get that, if $T<T_{0}$ then $\eta(T)=0$, and hence $r(T)=0$.

For the second case we study how the devil's staircase $r(T)$ behaves when $T \rightarrow 0$. Note that, when $r(T)<1$, this one coincides with the rotation number of the periodic orbits found when varying $T$ (see Remark 3.3).

Using that $\lim _{T \rightarrow 0} \eta(T)=0$, we get that, for any $T$ small enough, we can find $n$ large enough such that

$$
\frac{1}{n+1} \leq \eta(T) \leq \frac{1}{n}
$$

Hence, as $1 / n-1 /(n+1) \rightarrow 0$, it is enough to study how the steps given by the rotation numbers of the form $1 / n$ behave. Taking into account that the symbolic dynamics is organized by a Farey tree structure (a one to one mapping with the rotation numbers), this rotation numbers are associated with periodic orbits with symbolic sequences of the form $\mathcal{L}^{n} \mathcal{R}$. These periodic orbits are characterized by exhibiting one spike after $n$ iterations of the stroboscopic map, 
and are determined by the equations

$$
\begin{aligned}
\varphi\left(d T ; \bar{x}_{\mathcal{L}^{n} \mathcal{R}} ; A\right) & =x_{1} \\
\varphi\left(T-d T ; x_{1} ; 0\right) & =x_{1}^{\prime} \\
\varphi\left(d T ; x_{1}^{\prime} ; A\right) & =x_{2} \\
\varphi\left(T-d T ; x_{2} ; 0\right) & =x_{2}^{\prime} \\
\vdots & \\
\varphi\left(t^{\prime} ; x_{n}^{\prime} ; A\right) & =\theta \\
\varphi\left(d T-t^{\prime} ; 0 ; A\right) & =x_{n+1} \\
\varphi\left(T-d T ; x_{n+1} ; 0\right) & =\bar{x}_{\mathcal{L}^{n} \mathcal{R}},
\end{aligned}
$$

where $\varphi(t ; x ; A)$ is the flow associated with system $\dot{x}=f(x)+A$ and $\bar{x}_{\mathcal{L}^{n} \mathcal{R}}$ is the initial condition for the $\mathcal{L}^{n} \mathcal{R}$ periodic orbit for $t_{0}=0$. From the two last equations, we get that $\lim _{T \rightarrow 0} \bar{x}_{\mathcal{L}^{n} \mathcal{R}}=0$.

After applying a time rescaling, the original system (2.1) and its averaged version become

$$
\begin{aligned}
& \dot{x}=T(f(x)+\tilde{I}(t)) \\
& \dot{x}=T(f(x)+A d),
\end{aligned}
$$

where $\tilde{I}(t)$ is now 1-periodic. We now consider solutions of systems 3.15 and $(3.16$ with $O(T)$ close initial conditions. The averaging theorem of Bogoliubov and Mitropolski BM61 tells us that, if $T$ is small enough, then such solutions remain $O(T)$-close for a $t \sim 1 / T$ time scale provided that they have not reached the threshold. Note that the result given in BM61 applies because it does not require continuity in $t$ but boundedness and Lipschitz in $x$.

Hence, letting $\hat{\varphi}(t ; x)$ be the flow of the averaged system $(3.13)$, if $T$ is small enough we have that

$$
\varphi(T-d T ; \varphi(d T ; x ; A) ; 0)=\hat{\varphi}(T ; x)+O(T) .
$$

Hence, as long as the threshold is not reached, we can approximate the real flow by the averaged one. Using that $\bar{x}_{\mathcal{L}^{n} \mathcal{R}} \rightarrow 0$ when $T \rightarrow 0$, the time taken by the real flow to reach the threshold $x=\theta$ from $x=\bar{x}_{\mathcal{L}^{n} \mathcal{R}}$ approaches $\hat{\delta}$ :

$$
n T+t^{\prime} \rightarrow \hat{\delta}
$$

Hence, as $t^{\prime}<d T, n$ grows like $\hat{\delta} / T$ when $T \rightarrow 0$ and thus

$$
\lim _{T \rightarrow 0} r(T)=\lim _{T \rightarrow 0} \frac{1}{n T}=\frac{1}{\hat{\delta}} .
$$

Remark 3.8. Note that equation (3.14 makes sense only if the averaged system (3.13) has a stable critical point above the threshold, which occurs if $A d>$ $Q_{c}$. This occurs only in the permanent-spiking region. 
Remark 3.9. From Proposition 3.2 we get that the value of the firing-rate for small frequencies depends on the released dose Ad, the average of $I(t)$. However, for large values of $T$ (Proposition 3.1), it depends explicitly on $A$ and $d$.

\subsection{Optimization of the firing-rate}

As shown in Corollary 3.2 the firing-rate as a function of $T$, the period of the forcing $I(t)$, follows a devil's staircase with monotonically decreasing pieces (see Figure 2.1). This occurs for most values of $T$ except, possibly, in a bounded set, for which the firing-rate is an increasing function. Each of the pieces forming the devil's staircase occurs in a $T$-interval, $\left[T_{\sigma}^{\mathcal{R}}, T_{\sigma}^{\mathcal{L}}\right]$, for which a unique periodic orbit $\sigma$ exists. Hence, the firing-rate exhibits local maxima at $T=T_{\sigma}^{\mathcal{R}}$, and local minima at $T=T_{\sigma}^{\mathcal{L}}$. As a consequence of this there exists an infinite number of local minima and maxima at any interval of the form $\left[T_{\sigma}^{\mathcal{L}}, T_{\gamma}^{\mathcal{R}}\right]$, with $\sigma$ and $\gamma$ meaning different periodic orbits.

Of particular interest is when $\sigma$ and $\gamma$ are consecutive fixed points (spiking $n$ and $n+1$ times), because they occupy the largest regions in parameter space and their rotation numbers bound the ones of the periodic orbits, given by alternation of $n$ and $n+1$ spikes. Restricting to this case, we consider the firingrate in the frequency range corresponding to $\left[T_{n}^{\mathcal{L}}, T_{n+1}^{\mathcal{L}}\right]$. We will prove that the firing-rate follows a devil's staircase with monotonically decreasing steps but whose envelope is bell shaped; that is, it increases from $T_{n}^{\mathcal{L}}$ to $T_{n+1}^{\mathcal{R}}$, where it exhibits an absolute maximum, and then decreases to $T_{n+1}^{\mathcal{L}}$ (see fig. 3.8). Note that the bifurcation values $T_{n}^{\mathcal{L}}, T_{n+1}^{\mathcal{R}}$ and $T_{n+1}^{\mathcal{L}}$ can be easily found numerically (by solving equations (3.1) and (3.2) for $T$ ) and that the values of the firingrate at these values become $n / T_{n}^{\mathcal{L}},(n+1) / T_{n+1}^{\mathcal{R}}$ and $(n+1) / T_{n+1}^{\mathcal{L}}$, respectively. In real applications one is usually restricted to a bounded range of realistic frequencies for which one observes an absolute maximum of the firing rate (see for example [KJSC97, $\left.\mathrm{DHO}^{+} 89\right]$ ). Hence, this approach could be applied to properly tune system parameters in order to make the model exhibit such a behavior for the desired values of $T$.

We now investigate the optimization of the firing-rate in the whole range of periods, $(0, \infty)$.

Due to the fact that the firing-rate is bounded and continuous for $T>0$, it must exhibit a global maximum provided that it is increasing for $T=0^{+}$. From the argument above, it must occur at some value of the form $T_{\sigma}^{\mathcal{R}}$, respectively, for some periodic orbit $\sigma$. The next result tells us that, in general, this periodic orbit will be the $T$-periodic orbit spiking once per period.

Proposition 3.5. Let $(d, A)$ be in the spiking region (see Remark 3.7), and let $T_{1}^{\mathcal{R}}$ and $T_{1}^{\mathcal{L}}$ be the values of $T$ for which the periodic orbit spiking once per period undergoes right and left border collision. Then, there exists some $\gamma>0$ such that, if $T_{1}^{\mathcal{R}}>\gamma$ then the firing-rate $r(T)$ has global maximum at $T=T_{1}^{\mathcal{R}}$.

Proof. Let $T_{n}^{\mathcal{R}, \mathcal{L}}$ be the values of $T$ for which a $T$-periodic orbit spiking $n$ times undergoes border collision bifurcation on the right and left, respectively. As we know, the firing-number is a monotonically increasing function from $T_{n}^{\mathcal{L}}$ to 


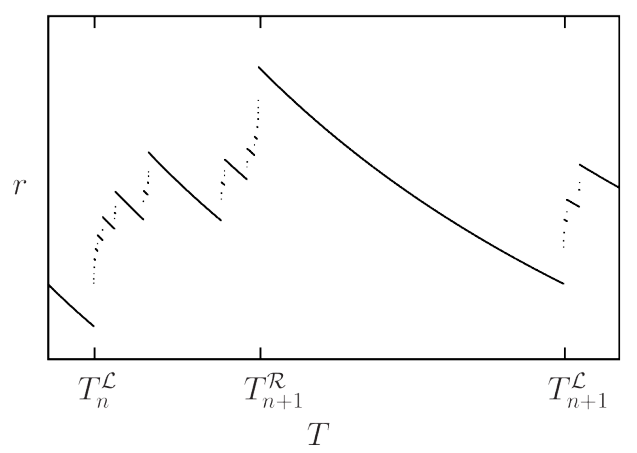

Figure 3.8: Typical response of the firing-rate between two consecutive fixed points. Its envelope is bell shaped, exhibiting a maximum at $T=T_{n+1}^{\mathcal{R}}$.

$T_{n+1}^{\mathcal{R}}$, for any $n$. Hence, the maximum must occur for some $T_{n}^{\mathcal{R}}$, right border collision bifurcation of the $T$-periodic orbit spiking $n$ times (see Figure 3.3(a) for $n=2$ ).

Taking into account relation 2.10 and recalling that the firing number for such orbits is $n$, the number of spikes, it will be enough to show that

$$
\frac{1}{T_{1}^{\mathcal{R}}}>\frac{2}{T_{2}^{\mathcal{R}}}>\frac{3}{T_{3}^{\mathcal{R}}} \ldots
$$

if $T_{1}^{\mathcal{R}}$ is large enough in order to see that this periodic orbit spikes once per period.

Let $\varphi\left(t ; x_{0} ; A\right)$ and $\delta>0$ be as in Proposition 3.3 , and let $\bar{x}_{n}$ be the fixed point of the stroboscopic map leading to the $T$-periodic orbit spiking $n$ times. Then $T_{n}^{\mathcal{R}}$ is determined by the following equations (see Figure 3.3(a) for $n=2$ )

$$
\begin{aligned}
\varphi\left(t_{n} ; \bar{x}_{n} ; A\right) & =\theta \\
\varphi\left(T_{n}^{\mathcal{R}}-d T_{n}^{\mathcal{R}} ; 0 ; 0\right) & =\bar{x}_{n} \\
t_{n}+(n-1) \delta & =d T_{n}^{\mathcal{R}} .
\end{aligned}
$$

As $T_{n+1}^{\mathcal{R}}>T_{n}^{\mathcal{R}}$, recalling that the flow $\varphi\left(t ; x_{0} ; 0\right)$ is exponentially attracted by the equilibrium point $\bar{x}_{n}$, from the second equation it comes that, at the moment of the bifurcation

$$
\begin{aligned}
\bar{x}_{n}<\bar{x}_{n+1} & \rightarrow \bar{x} \\
t_{n}>t_{n+1} & \rightarrow \bar{t},
\end{aligned}
$$

where $\bar{t}$ is the smallest such that $\varphi(\bar{t} ; \bar{x} ; A)=\theta$.

As these series converge exponentially (due to the hyperbolicity of $\bar{x}$ ), we have that there exists some $N>0,0<\lambda<1$ and $K>0$ such that

$$
n \underbrace{\left(t_{n}-t_{n+1}\right)}_{<K \lambda^{n}}+t_{n} \rightarrow \bar{t}, n>N
$$


Assuming $n>N$ large enough and using that $\delta>\bar{t}$ we get

$$
\begin{aligned}
\delta>n\left(t_{n}-t_{n+1}\right)+t_{n} & \Longleftrightarrow n t_{n+1}+\delta>(n+1) t_{n} \\
& \Longleftrightarrow n \overbrace{\left(t_{n+1}+n \delta\right)}^{T_{n+1}^{\mathcal{R}}}>(n+1) \overbrace{\left(t_{n}+(n-1) \delta\right)}^{T_{n}^{\mathcal{R}}} \\
& \Longleftrightarrow \frac{n}{T_{n}^{\mathcal{R}}}>\frac{n+1}{T_{n+1}^{\mathcal{R}}} .
\end{aligned}
$$

In particular, if $T_{1}^{\mathcal{R}}$ is large enough, $\bar{x}_{1}$ is close enough to $\bar{x}$ to fulfill (3.17) and $(3.18)$ for $n=1$.

Remark 3.10. Note that, $T_{1}^{\mathcal{R}}$ will be large enough if $\bar{x}$ is attracting enough.

Remark 3.11. Arguing similarly, the global minimum will be the minimum of 0 (if $(d, A)$ belongs to the conditional spiking region), $1 / \hat{\delta}$ (Proposition 3.4) and $1 / T_{1}^{\mathcal{L}}$. Note that if the minimum corresponds to $1 / \hat{\delta}$, then it technically does not exist, as $T=0$ is excluded from the domain.

Corollary 3.4. For a given system (2.1)-(2.2), the globally maximal firing-rate is achieved with the combination of period $T$, dose $Q$, amplitude $A$ and duty cycle $d$ such that the straight line $1 / A=1 / Q d$ is tangent to the bifurcation curve $A=A_{n}^{\mathcal{R}}(d)$ for the smallest possible value of $T$.

\section{Example}

In this section we use the results presented so far to study the behavior of the firing-rate under frequency variation for different configurations. For the sake of simplicity, we choose to study such configurations for a linear system, as it will permit us to compute explicitly the quantities involved in the results of section 3.2. However, we emphasize that these quantities are straight forward to compute numerically for other type of systems for which conditions H.1-H.2 hold.

\subsection{Linear integrate and fire model}

Let

$$
f(x)=a x+b .
$$

In order to satisfy conditions H.1-H.2, we require that $a<0$ and $\bar{x}=-b / a \in$ $(0, \theta)$, where $x=\theta$ is the threshold of the integrate and fire system (2.1)-(2.2).

For system 2.1)-2.2 - (4.1), the critical dose 2.11) becomes

$$
Q_{c}=-(a \theta+b),
$$

which is the minimal amplitude of the pulse (2.3) for which the system (2.1)(2.2)-4.1) can exhibit spikes. 
The linearity of the system permits us to also explicitly compute the quantity $\delta$ involved in Proposition 3.3 .

$$
\delta=\frac{1}{a} \ln \left(\frac{\theta a}{b+A}+1\right) .
$$

The averaged version of system (2.1)-(2.2)-(4.1) becomes $\dot{x}=f(x)+A d$, for which we can also explicitly compute the quantity $\hat{\delta}$ involved in Proposition 3.4

$$
\hat{\delta}=\frac{1}{a} \ln \left(\frac{\theta a}{b+A d}+1\right) .
$$

In Figures 4.1 and 4.2 we show the bifurcation scenario in the $d \times 1 / A$ parameter space for different values of $T$. As mentioned in Remark 3.5 , for some values of $T$ the stroboscopic map $\mathfrak{s}$ may lose contractiveness in the domain $\left[\Sigma_{n}, \theta\right]$. When this occurs, the rotation number (and hence the firing number and firing-rate) do not follow a devil's staircase but a monotonically increasing continuous function.

As shown in GKC13, for the linear case the contracting condition becomes

$$
\begin{aligned}
& F(A, T, d)=\left|\left[\Sigma_{n}, \theta\right]\right|-\left|\mathfrak{s}\left(\left[\Sigma_{n}, \theta\right]\right)\right|=\theta-\Sigma_{n}-\left(\mathfrak{s}(\theta)-\mathfrak{s}\left(\Sigma_{n}\right)\right) \\
& =\theta-\frac{b+A}{a}\left(e^{a(n \delta-d T)}-e^{a(T-(n-1) \delta)}+e^{a T(1-d)}-1\right) .
\end{aligned}
$$

On one hand, one sees that $F$ is a monotonically decreasing function of $A$. On the other hand, when the fixed point $\bar{x}_{1}$ undergoes a right border collision $\left(A=A_{1}^{\mathcal{R}}\right) \mathfrak{s}$ is contractive in the whole domain $[0, \theta]$. Therefore, if it exists, the region where the rotation number is not a devil's staircase is bounded in the parameter space $d \times 1 / A$ between the curves given by $A=A_{0}(d)$ and $A=A_{1}^{\mathcal{R}}(d)$. Hence, below the curve given by $A=A_{1}^{\mathcal{R}}$ only devil's staircases given by the period adding bifurcation structures can exist. See GKC13 for more details.

As predicted by Proposition 3.2, when $T \rightarrow 0$ the first bifurcation curve, $A_{0}(d)$, tends to be the straight line $1 / A=1 / Q_{c} d$, and the rest of bifurcation curves accumulate at $1 / A=0$ (see Figure 4.1).

By contrast, when $T \rightarrow \infty$, all bifurcation curves accumulate at the horizontal curve $1 / A=1 / Q_{c}$, as predicted by Proposition 3.1 (see Figure 4.2.

We are now interested in studying the firing-rate $r$ 2.10 under frequency variation. However, when varying the period of the pulse $(2.3)$, we will restrict ourselves to pulses with constant average (constant released dose or energy) $Q$ given in equation 2.4.

Obviously, the output of the system will be sensitive to variations of the injected energy (dose). Hence, in order to perform an analysis based exclusively on frequency variation we will be interested in the variation of the frequency of the stimulus while keeping the dose constant (dose conservation).

Note that points in the parameter space $d \times 1 / A$ with a fixed dose are located in the straight lines

$$
\frac{1}{A}=\frac{1}{Q} d .
$$




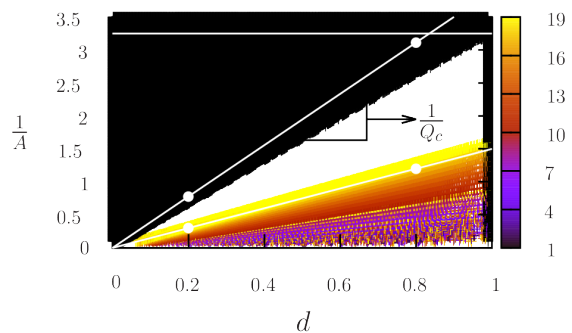

(a)

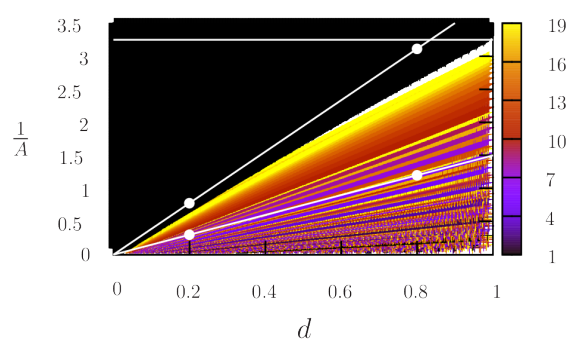

(c)

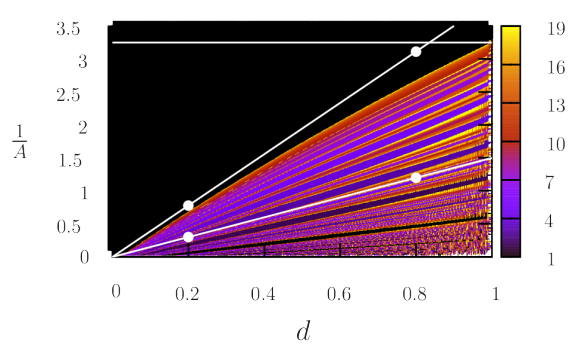

(e)

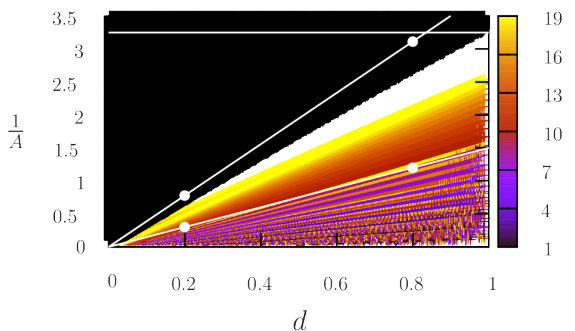

(b)

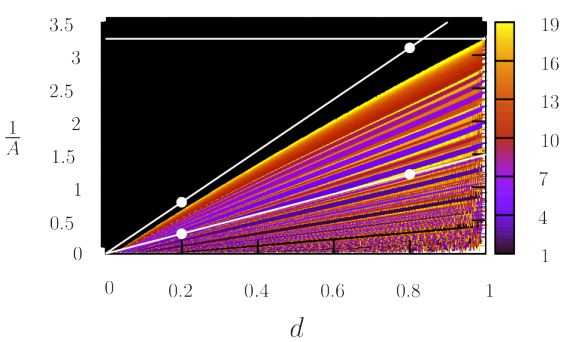

(d)

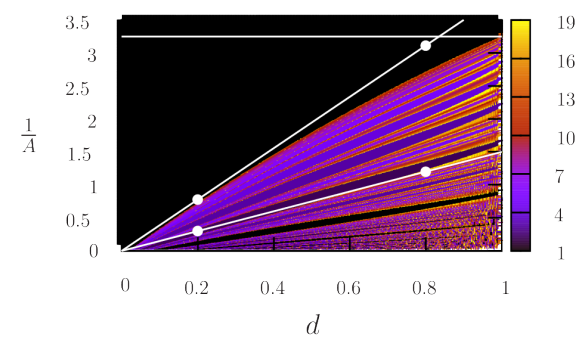

(f)

Figure 4.1: Bifurcation scenarios for $T=0.1$ (a), $T=0.2(\mathrm{~b}), T=0.3$ (c), $T=0.5(\mathrm{~d}), T=0.7$ (e) and $T=1$ (f), for $\theta=1, b=0.2, a=-0.5$. For clarity reasons only periods lower than 20 are shown; regions with higher periods are filled in white. The horizontal line $\left(1 / A=1 / Q_{c}\right)$ separates the non-spiking and the spiking regions. The straight line with slope $1 / Q_{c}$ labeled in (a) separates the permanent and conditional spiking regions. The white lines are given by parameters leading to the same dose: $A d=$ const. 


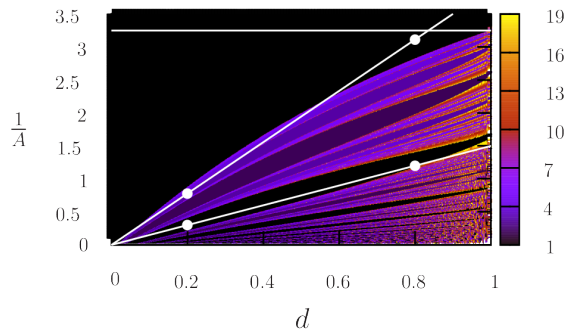

(a)

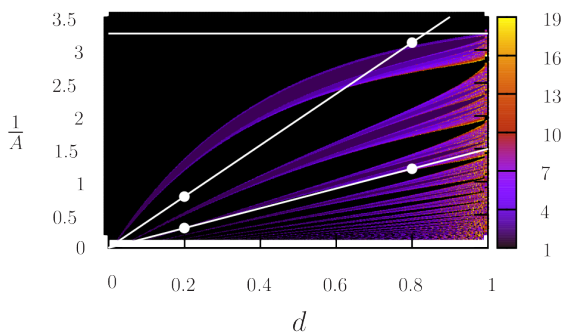

(c)

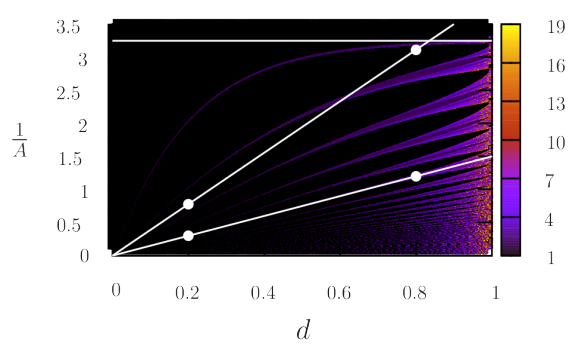

(e)

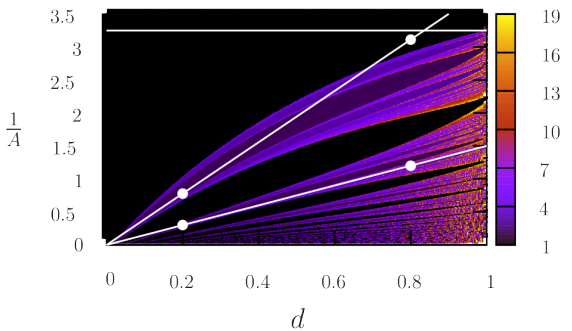

(b)

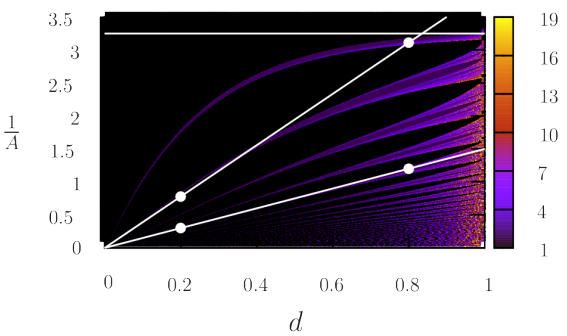

(d)

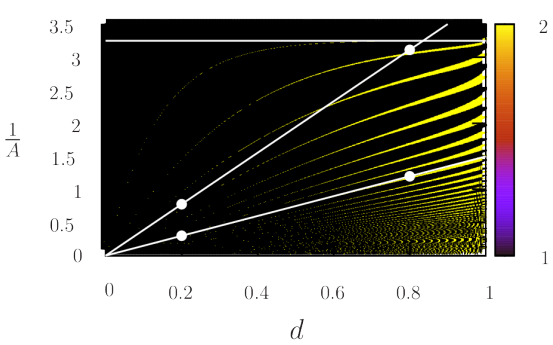

(f)

Figure 4.2: Bifurcation scenarios for $T=2$ (a), $T=3$ (b), $T=5$ (c), $T=8$ (d), $T=10$ (e) and $T=15$ (f). See caption in Fig. 4.1 for more details. In (f) only periods up to 2 are shown in order to improve comparison between (e) and (f). 
In Figures 4.1 and 4.2 we have highlighted parameter values associated to two different doses. These are given by two different white straight lines; the one with the larger slope $\left(Q<Q_{c}\right)$ is fully contained in the non-spiking region when $T$ small enough, while the other one is contained in the spiking region for all values of $T$, and they will lead to different qualitative responses.

Note that the dose conservation can be performed in three different ways in order to keep the quantity $Q=A d$ constant. In the first one one varies the duration of the impulse $\Delta=d T$ as the period $T$ of the periodic input $I(t)$ varies, while its amplitude $A$ is kept constant. This is done by keeping the duty cycle $d$ constant.

In the second one, the duration of the pulse is fixed, and one varies its amplitude when $T$ is varied in order to keep constant the average of $I(t)$.

Of course, one can also simultaneously vary both magnitudes, giving rise to any different types of parametrizations with respect to $T$ of the straight lines corresponding to fixed dose.

In the next sections we separately study the first two cases.

\subsection{Fixed dose for constant impulse amplitude (width cor- rection)}

Taking into account that $1 / A=d / Q$, for a fixed value of the amplitude of the pulse it is enough to keep the duty cycle $d$ constant in order to obtain an input with constant dose $Q$. Hence, in this first approach, we just fix one point in the parameter space $(d, 1 / A)$ and vary $T$. This will allow us to directly apply the results shown in $\S 3.2$.

In Figure 4.3 we focus on two points in the parameter space located at the white straight line with lower slope in Figs. 4.1 and 4.2 (higher dose, $Q>Q_{c}$ ), and we show the firing-number, $\eta$, (left figures) and the firing-rate, $r$, (right figures) of the periodic orbits found when varying $T$.

As announced in Corollary 3.1, as $Q>Q_{c}$ these two points in the parameter space are located in the permanent-spiking region and, hence, as mentioned in Corollary 3.3, the firing-number tends to zero when $T \rightarrow 0$. However, as predicted by Proposition 3.4, the firing-rate fulfills

$$
\lim _{T \rightarrow 0} r(T)=\frac{1}{\hat{\delta}},
$$

with $1 / \hat{\delta}=0.58$ for the used parameter values. As noted in Remark 3.8 this value only depends on $Q$ and hence it is the same for all points with equal dose.

In Figure 4.4 we show a magnification of the firing rate for small values of $T$, where one can clearly see the structure given by the devil's staircase.

On the other hand, Proposition 3.3 provides the limiting value for the firingrate,

$$
\lim _{T \rightarrow \infty} r(T)=\frac{d}{\delta},
$$

where $\delta$ is given in 4.2 . Note that this quantity depends on $A$ and, hence, it is different for the two considered case although they correspond to inputs with 


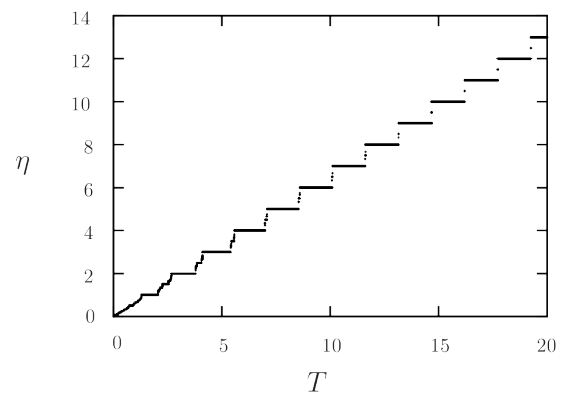

(a)

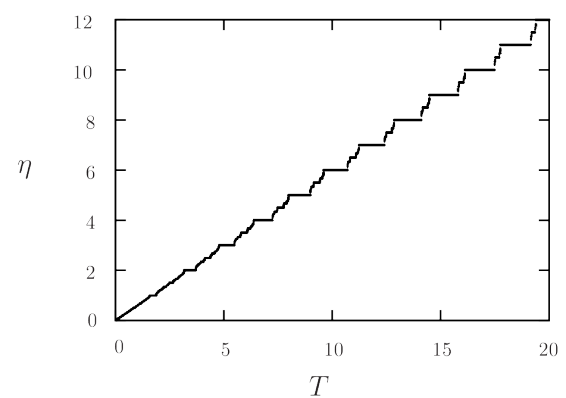

(c)

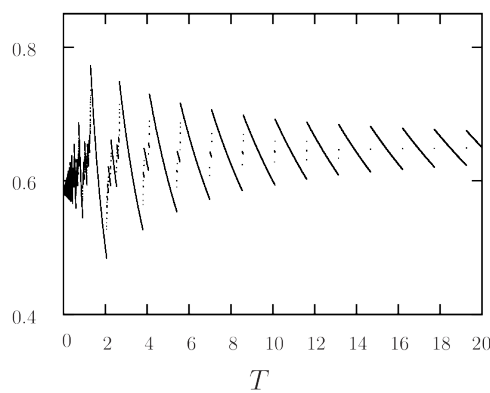

(b)

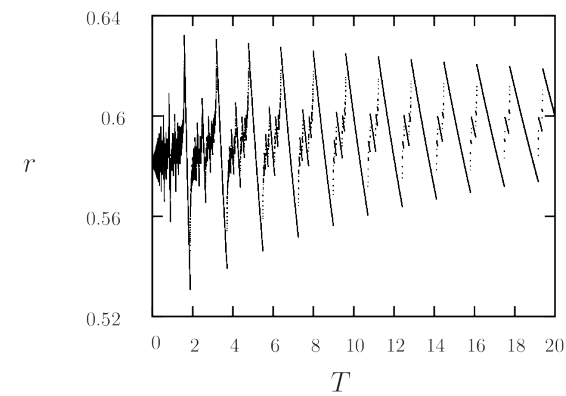

(d)

Figure 4.3: Firing-number $\eta(T)$ (left) and firing-rate $r=\eta(T) / T$ (right), under variation of $T$ for $Q=0.666$. (a) and (b) $d=0.2$ and $1 / A=0.3$. (c) and (d) $d=$ 0.8 and $1 / A=1.2$. The firing-rate follows a devil's staircase with monotonically decreasing pieces exhibiting a maximum at $T=T_{1}^{\mathcal{R}}$ and a minimum at $T=T_{1}^{\mathcal{L}}$. Parameters $a, b$ and $\theta$ are set as in Figure 4.1 .

the same average. For $(d, 1 / A)=(0.2,0.3)$ (Figure $4.3(\mathrm{~b}))$ we get $d / \delta=0.655$, and for $(d, 1 / A)=(0.8,1.2)$ we obtain $d / \delta=0.604$.

Finally, observe that the firing-rate possesses a global maximum and minimum at $T=T_{1}^{\mathcal{R}}$ and $T=T_{1}^{\mathcal{L}}$, respectively, as $\bar{x}$ is attracting enough.

We now focus on two different inputs with average lower than the critical dose. In Figure 4.5 we show the same results for the two points labeled in Figures 4.1 and 4.2 located on the white straight line with higher slope (lower dose). For large values of $T$ the firing-rate shows the same behavior as before with limiting values $d / \delta=0.244$ (Figure $4.5(\mathrm{~b})$ ) and $d / \delta=0.125$ (Figure 4.5 (d)). However, as predicted in Corollary 3.3 , unlike in the previous case, as these two points are now located in the conditional-spiking region, there exists some values of $T$ below which the firing-rates vanish.

Note that, as in the previous case, the firing-rate exhibits a global maximum at $T=T_{1}^{\mathcal{R}}$. However, the global minimum becomes now 0 for all $0<T<T_{0}$, 


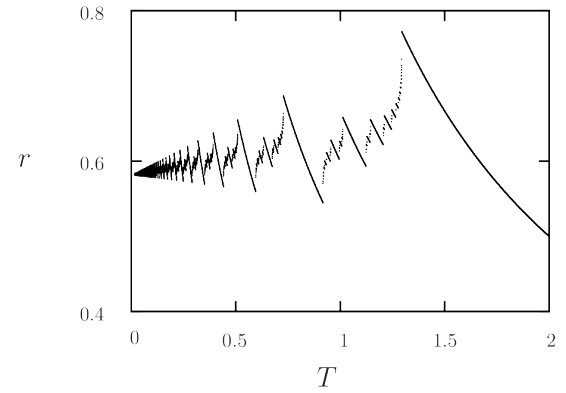

(a)

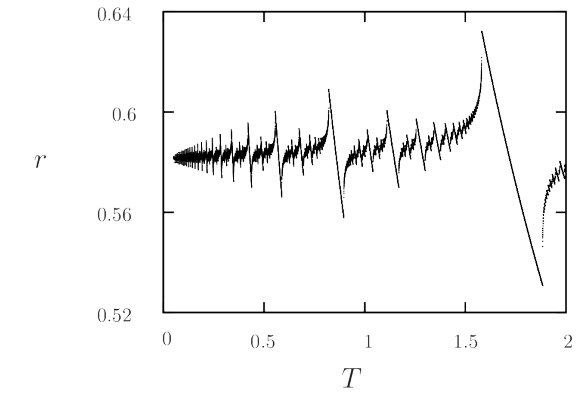

(b)

Figure 4.4: Magnification of Figures 4.3 (b) and (d).

as $(d, A)$ belongs to the conditional spiking region (see Remark 3.11).

\subsection{Fixed dose for fixed pulse duration (amplitude correc- tion)}

We now fix the duration of the pulse $\Delta$ and perform the dose conservation by properly modifying its amplitude. This is done by varying the parameters $d$ and $A$ along straight lines in the parameter space $d \times 1 / A$ parametrized by $T$,

$$
\left(d, \frac{1}{A}\right)=\left(\frac{\Delta}{T}, \frac{\Delta}{Q T}\right) .
$$

Note that, with this approach, it is not possible to analyze the properties of the output when $T \rightarrow 0$, since its minimal value is $T=\Delta$. Varying $T$ from $\Delta$ to $\infty$, one has to vary $(d, 1 / A)$ from $(1,1 / Q)$ to $(0,0)$ along a straight line with slope $1 / Q$ in order to keep the released dose constant.

Regarding the behavior of $r(T)$ when $T \rightarrow \infty$, we can use Proposition 3.3 From (4.3) we get $d=\Delta / T$ and $A=Q T / \Delta$, which, when combined with 4.2 and Proposition 3.3 gives us

$$
\lim _{T \rightarrow \infty} r(T)=\frac{Q}{\theta},
$$

independently of $\Delta$.

In Figure 4.6 we show the evolution of the firing-rate for an input with average greater than the critical dose, $Q_{c}$. Note that this leads to a broken devil's staircase, as it starts at $T=\Delta$. The behavior at $T \rightarrow \infty$ is the expected one.

In Figure 4.7 we show the same computation for a $Q<Q_{c}$. In this case, if $T$ is close enough to $\Delta$, equation 4.3 provides points located in the non-spiking 


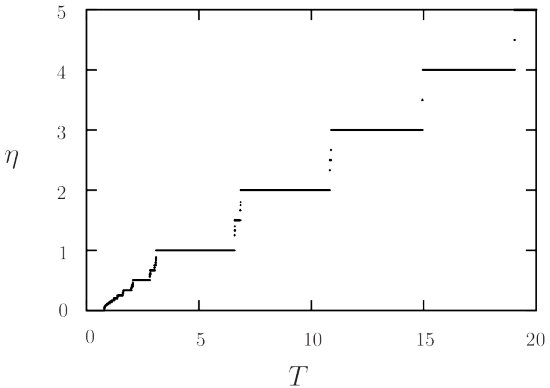

(a)

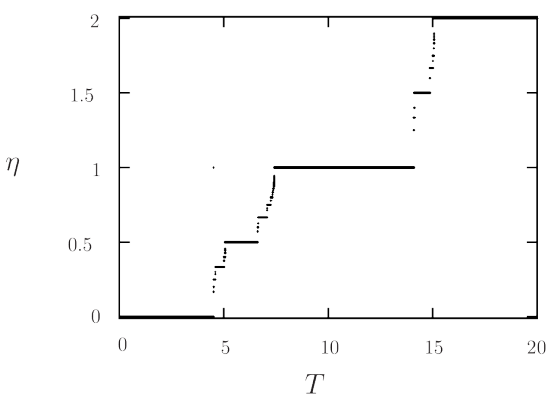

(c)

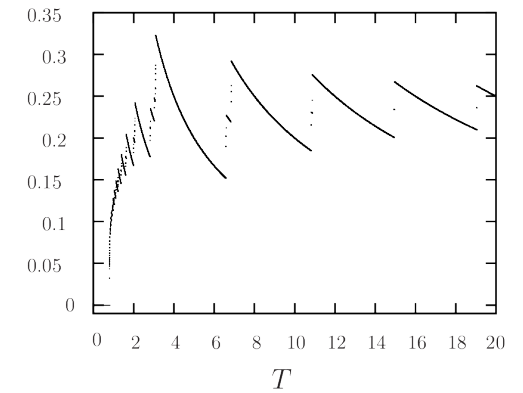

(b)

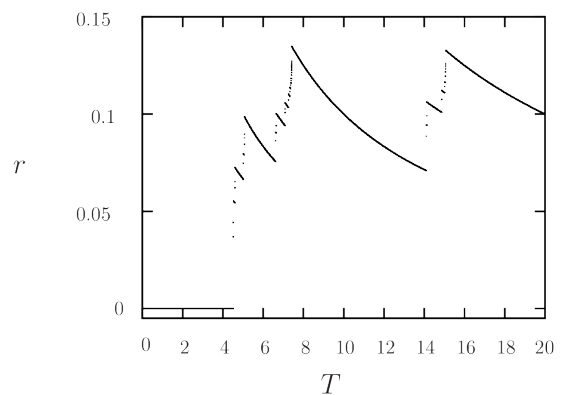

(d)

Figure 4.5: Same as Fig. 4.3 for $Q=0.257$. (a) and (b): $d=0.2$ and $1 / A=$ 0.777. (c) and (d): $d=0.81 / A=3.111$. As in the previous case, a global maximum occurs at $T=T_{1}^{\mathcal{R}}$; however, the global minimum is 0 for a whole range of small periods. 


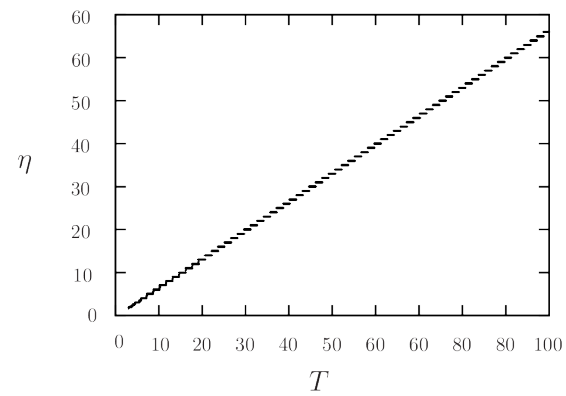

(a)

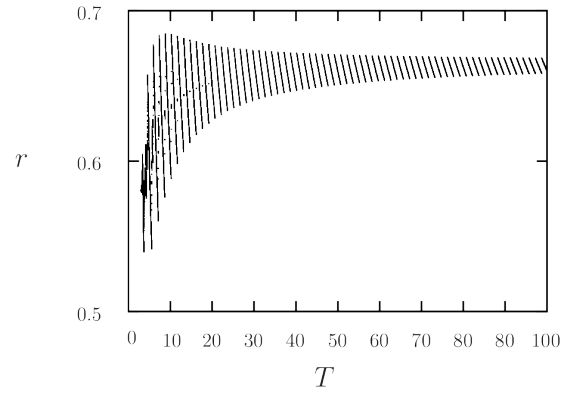

(b)

Figure 4.6: Firing-number (a) and firing-rate (b) for $Q=0.666$ using amplitude correction while keeping constant the duration of the pulse, $\Delta=3$.

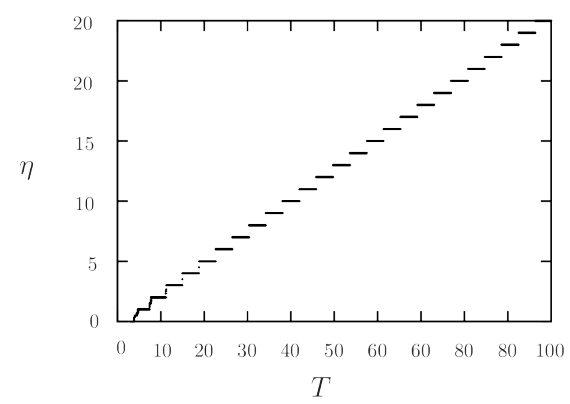

(a)

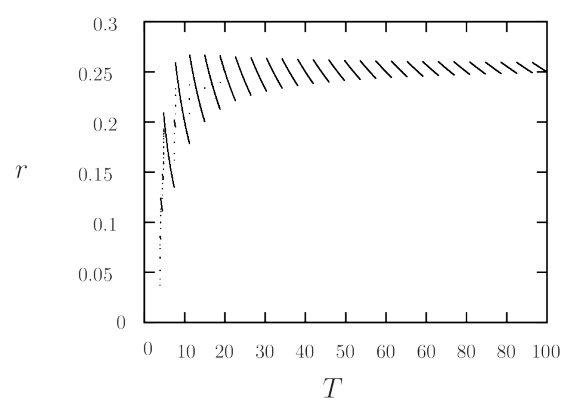

(b)

Figure 4.7: Firing-number (a) and firing-rate (b) for $Q=0.257$ using amplitude correction while keeping constant the duration of the pulse, $\Delta=3$.

region for which $r(T)=\eta(T)=0$. Although the points provided by 4.3 are never located in the permanent-spiking region, they are in the conditionalspiking region if $T$ is large enough. Hence, one starts observing spikes at some point.

Note that, unlike when fixing the dose by width correction, one always gets non zero spikes per period and non-zero firing-rates, at least for small enough frequencies. This is because, when following the straight lines 4.3 towards the origin one always enters the spiking regions.

\section{Conclusions}

In this paper we have considered a generic spiking model (integrate-and-fire-like system) with an attracting equilibrium point in the subthreshold regime forced 
by means of a pulsatile (square wave) periodic input. By contrast to the usual approach [KHR81, COS01, TB08, we consider the stroboscopic map instead of the Poincaré map onto the threshold. This Poincaré map becomes a regular map there where it is defined while the stroboscopic map is discontinuous. However, as shown in GKC13, this becomes indeed an advantage, as this type of maps are well understood map by the piecewise-smooth community (see AGGK for a recent survey).

As shown in GKC13] the system exhibits spiking dynamics organized in rich bifurcation structures in the parameter space formed by the amplitude and duty cycle of the forcing pulse. These bifurcations and the associated symbolic dynamics completely explain relevant features of this type of excitable systems, like the firing-rate. In this work, we have studied how these bifurcation structures, and dynamical properties associated with them, vary when the period of the forcing is varied while keeping the injected dose (input average) constant. We have given special interest to the asymptotic firing-rate (average number of spikes per unit time), which turns out to follow a devil's staircase (a fractal structure) with monotonically decreasing steps. In particular, we have precisely characterized its global maximum in the whole frequency domain as well each local maxima. If we consider specific ranges of frequency whose bounds correspond to the frequencies eliciting the subsequent local minima, the response can be decomposed in a repetitive structure with a non-monotonic, bell-shaped pattern and global maximum.

\section{References}

[AGGK] Ll. Alsedà, J.M. Gambaudo, A. Granados, and M. Krupa. Period adding and incrementing in one-dimensional discontinuous maps: theory and applications. In preparation.

[BM61] N.N. Bogoliubov and Y.A. Mitropolski. Asymptotic methods in the theory of non-linear oscillations. Gordon and Breach, 1961.

[CB99] S. Coombes and P.C. Bressloff. Mode locking and Arnold tongues in integrate-and-fire neural oscillators. Phys. Rev E., 60:2086-2096, 1999.

[CO00] S. Coombes and A. H. Osbaldestin. Period-adding bifurcations and chaos in a periodically stimulated excitable neural relaxation oscillator. Phys. Rev. E, 62:4057-4066, 2000.

[Coo01] S. Coombes. Phase-locking in networks of pulse-coupled mckean relaxation oscillators. Physica D, 2820:1-16, 2001.

[COS01] S. Coombes, M. Owen, and G.D. Smith. Mode locking in a periodically forced integrate-and-fire-or-burst neuron model. Phys. Rev E., 64:041914, 2001. 
[CTW12] S. Coombes, R. Thul, and K.C.A Wedgwood. Nonsmooth dynamics in spiking neuron models. Physica D, 241:2042-2057, 2012.

$\left[\mathrm{DHO}^{+} 89\right]$ A.C. Dalkin, D.J. Haisenleder, G.A. Ortolano, T.R. Ellis, and J.C. Marshall. The frequency of gonadotropin-releasing-hormone stimulation differentially regulates gonadotropin subunit messenger ribonucleic acid expression. Endocrinology, 125:917-924, 1989.

[FG11] J.G. Freire and J.A.C. Gallas. Stern-brocot trees in cascades of mixed-mode oscillations and canards in the extended bonhoeffervan der pol and the fitzhugh-nagumo models of excitable systems. Phys. Lett. A, 375:1097-1103, 2011.

[GGT84] J.M. Gambaudo, P. Glendinning, and C. Tresser. Collage de cycles et suites de Farey. C. R. Acad. Sc. Paris, série I, 299:711-714, 1984.

[GIT84] J.M. Gambaudo, O.Lanford III, and C. Tresser. Dynamique symbolique des rotations. C. R. Acad. Sc. Paris, série I, 299:823-826, 1984 .

[GKC13] A. Granados, M. Krupa, and F. Clément. Border collision bifurcations of stroboscopic maps in periodically driven spiking models. Preprint available at http://arxiv.org/abs/1310.1054, 2013.

$\left[\mathrm{JMB}^{+} 13\right]$ N.D Jimenez, S. Mihalas, R. Brown, E. Niebur, and J. Rubin. Locally contractive dynamics in generalized integrate-and-fire neurons. SIAM J. Appl. Dyn. Syst. (SIADS), 12:1474-1514, 2013.

[KHR81] J.P. Keener, F.C Hoppensteadt, and J. Rinzel. Integrate-and-fire models of nerve membrane response to oscillatory input. SIAM J. Appl. Dyn. Syst. (SIADS), 41:503-517, 1981.

[KJSC97] U.B. Kaiser, A. Jakubowiak, A. Steinberger, and W.W. Chin. Differential effects of gonadotropin-releasing hormone $(\mathrm{GnRH})$ pulse frequency on gonadotropin subunit and $\mathrm{GnRH}$ receptor messenger ribonucleic acid levels in vitro. Endocrinology, 138:1224-1231, 1997.

[LC05] C.R. Laing and S. Coombes. Mode locking in a periodically forced "ghostbursting" neuron model. Int. J. Bif. Chaos, 15:1433, 2005.

[MHR12] X. Meng, G. Huguet, and J. Rinzel. Type III excitability, slope sensitivity and coincidence detection. Disc. Cont. Dyn. Syst., 32:2720$2757,2012$.

[TB08] J. Touboul and R. Brette. Dynamics and bifurcations of the adaptive exponential integrate-and-fire model. Biol. Cybernet, 99:319-334, 2008.

[TB09] J. Touboul and R. Brette. Spiking dynamics of bidimensional integrate-and-fire neurons. SIAM J. Appl. Dyn. Syst. (SIADS), 4:1462-1506, 2009. 